\title{
Near-field relaxation of a quantum emitter to two-dimensional semiconductors: Surface dissipation and exciton polaritons
}

\author{
Vasilios D. Karanikolas, ' Cristian A. Marocico, Paul R. Eastham, and A. Louise Bradley \\ Photonics Group, School of Physics and CRANN, Trinity College Dublin, College Green, Dublin 2, Ireland \\ (Received 7 August 2016; revised manuscript received 11 October 2016; published 11 November 2016)
}

\begin{abstract}
The total spontaneous emission rate of a quantum emitter in the presence of an infinite $\mathrm{MoS}_{2}$ monolayer is enhanced by several orders of magnitude, compared to its free-space value, due to the excitation of surface exciton polariton modes and lossy modes. The spectral and distance dependence of the spontaneous emission rate are analyzed and the lossy surface wave, surface exciton polariton mode and radiative contributions are identified. The transverse magnetic and transverse electric exciton polariton modes can be excited for different emission frequencies of the quantum emitter, and their contributions to the total spontaneous emission rate are different. To calculate these different decay rates we use the non-Hermitian description of light-matter interactions, employing a Green's tensor formalism. The distance dependence follows different trends depending on the emission energy of the quantum emitter. For the case of the lossy surface waves, the distance dependence follows a $z^{-n}, n=2,3,4$, trend. When transverse magnetic exciton polariton modes are excited, they dominate and characterize the distance dependence of the spontaneous emission rate of a quantum emitter in the presence of the $\mathrm{MoS}_{2}$ layers. The interaction between a quantum emitter and a $\mathrm{MoS}_{2}$ superlattice is investigated, and we observe a splitting of the modes supported by the superlattice. Moreover, a blueshift of the peak values of the spontaneous emission rate of a quantum emitter is observed as the number of layers is increased. The field distribution profiles, created by a quantum emitter, are used to explain this behavior.
\end{abstract}

DOI: 10.1103/PhysRevB.94.195418

\section{INTRODUCTION}

The emission properties of quantum emitters are modified by their environment [1]. In particular, through excitation of surface plasmon modes, the spontaneous emission rate of a quantum emitter (QE) can be enhanced by several orders of magnitude compared with its free-space value [2,3]. Surface plasmon polaritons are collective oscillations of electrons and the electromagnetic field that are excited at the interface between a dielectric and a conductor, and they are confined at this interface and propagate along it. Noble metals, such as Au and $\mathrm{Ag}$, are typically used as plasmonic materials. However, the main disadvantage of using noble metals is the fact that they have high losses in the optical region of the spectrum [4]. As an alternative for materials supporting surface plasmon modes, but with lower losses, graphene can be considered [5,6]. Graphene is a zero direct band-gap two-dimensional material of great potential and with good mechanical capabilities [7]. However, it also has a disadvantage, since it exhibits no plasmonic response in the visible part of the spectrum and acts only as a quencher when interacting with quantum emitters emitting in the visible part of the spectrum [8,9].

In addition to surface plasmon modes, there are other surface modes such as phonon and exciton polariton $[10,11]$ modes. In previous years, a new family of two-dimensional materials, the transition metal dichalconides (TMD), such as $\mathrm{MoS}_{2}, \mathrm{SnS}_{2}$ and $\mathrm{WeS}_{2}$, have been the subject of intense theoretical [12-14] and experimental investigations [15,16]. These materials are direct band-gap semiconductors, with the conduction and valence band edges at the doubly degenerate corners ( $\pm \mathbf{K}$ points) of the hexagonal Brillouin zone, and can have relatively high absorption and intense photoluminescence

*karanikv@tcd.ie
$[17,18]$. We calculate the spontaneous emission rate for a $\mathrm{QE}$ above a $\mathrm{MoS}_{2}$ layer and find that the spontaneous emission rate is enhanced by many orders of magnitude. We trace this effect to the near-field energy transfer from the QE to the surface exciton polariton. In addition to implications for energy transfer applications, such as photodetectors [19], photovoltaic [20], and light emitting devices [21-23], our results show that low-dimensional materials can be used to study polaritons and exciton-photon coupling phenomena without requiring a microcavity [24]. Herein we demonstrate that $\mathrm{MoS}_{2}$ monolayers can support surface exciton polariton modes, and their influence on the optical properties of QEs is substantial.

The interaction between quantum emitters (QEs) and multilayers of TMD materials is of particular experimental interest. Many applications can benefit from manipulating these interactions, such as photodetectors [19], electronic [25], photovoltaic [20], and light emitting devices [21-23]. Investigating the spectral and distance dependence of the interactions between QEs and TMD layers or monolayers is of absolute importance for such applications. Various experimental studies have been performed regarding the investigation of such interactions, and they report contradicting results concerning the power law followed by the interaction distance between the QE-TMD layers, where different QEs are considered for each case [26-31]. A systematic analysis is needed to account for the spectral and distance dependence of the QE-TMD layer interaction. Here we focus on material parameters describing the semiconducting behavior of $\mathrm{MoS}_{2}$, through the exciton energies and damping parameters [17,24,32].

We find that transverse electric (TE) and transverse magnetic (TM) exciton polariton modes are supported by a $\mathrm{MoS}_{2}$ layer, Fig. 3. The propagation length and penetration depth of these modes are investigated. The SE rate of the QE is enhanced several orders of magnitude for emission energies 
close to the exciton energies, especially when the TM exciton polariton modes are excited, in the presence of a single $\mathrm{MoS}_{2}$ layer, see Fig. 6. The different contributions to the total SE rate are presented: the lossy surface wave (LSW), TE and TM exciton polariton modes and radiative emission contribution, for different $\mathrm{QE}-\mathrm{MoS}_{2}$ separations and emission energies of the QE.

Additional physics appears as one goes beyond the single layer structure to multilayers. In particular, we show that the electromagnetic coupling between the layers splits the degeneracy of the exciton polariton modes, even in the absence of direct electronic coupling. We find, see Fig. 7, that the electromagnetic coupling between the layers leads to a blueshift in the peak of the spontaneous emission rate with an increasing number of layers. This may provide an explanation for the different dependences of the emission rate with the layer number observed in experiments [29,30].

In Sec. II we introduce the mathematical method for studying the $\mathrm{QE}-\mathrm{MoS}_{2}$ structure. The QE is described as a two-level system, and the Green's tensor formalism is used to describe the light-matter interaction in the non-Hermitian description of quantum electrodynamics, Sec. II A. The optical response of the $\mathrm{MoS}_{2}$ layer is modeled by the surface conductivity, Sec. II B. In Sec. III we give the results. We start in Sec. III A by analyzing the surface exciton polariton when a single exciton resonance is considered in the surface conductivity. When two exciton resonances are considered, we see that two bands are formed, corresponding to the TE and TM exciton polariton modes, Sec. III A. The propagation length and penetration depth of the TE and TM exciton polariton modes are analyzed. In Sec. III B, the interaction between a $\mathrm{QE}$ and a free-standing $\mathrm{MoS}_{2}$ layer is considered. The spectral and distance dependence is analyzed, and the different contributions are studied. The LSW, TE, and TM exciton polariton modes and radiative emission contributions to the $\mathrm{SE}$ rate of a $\mathrm{QE}$, at different positions and emission energies, are presented. In Sec. III C we focus on the interaction between $\mathrm{QE}$ and $\mathrm{MoS}_{2}$ planar superlattices. We observe that the TE and TM exciton polariton modes bands are still split and that multibands are also formed, due to interlayer scattering. The SE peak of the QE is blueshifted, and the absolute value of the SE rate enhancement decreases. Finally, in Sec. IV we give some concluding results and future steps for research in the field.

\section{MATHEMATICAL METHODS}

\section{A. Spontaneous emission rate}

The quantum emitters $(\mathrm{QEs})$ considered in this paper are approximated as two-level systems. Various emitters, such as atoms, molecules, quantum dots and $\mathrm{NV}$ color centers, can be approximated in this way. The ground state of the QE is denoted as $|g\rangle$, and the excited state as $|e\rangle$. The transition frequencies from the excited to the ground state and the transition dipole matrix element are denoted as $\omega_{\mathrm{T}}$ and $\boldsymbol{\mu}$, respectively. The multipolar Hamiltonian is used to describe a QE interacting with the electromagnetic field $[33,34]$, and it has the form

$$
\begin{aligned}
\hat{H}=\hat{H}_{\mathrm{em}}= & \int \mathrm{d}^{3} r \int_{0}^{\infty} \mathrm{d} \omega \hbar \omega \hat{\mathbf{f}}^{\dagger}(\mathbf{r}, \omega) \cdot \hat{\mathbf{f}}(\mathbf{r}, \omega)+\hbar \omega_{T} \sigma^{+} \sigma^{-} \\
& -\int d \omega[\hat{\boldsymbol{\mu}} \cdot \hat{\mathbf{E}}(\mathbf{r}, \omega)+\text { H.c. }],
\end{aligned}
$$

where $\hat{\boldsymbol{\mu}}=\boldsymbol{\mu} \sigma^{+}+\boldsymbol{\mu}^{*} \sigma^{-}$is the transition dipole operator of the two level system, with $\boldsymbol{\mu}$ being the transition dipole moment of the system between its ground and excited states. The electric field operator has the form

$$
\hat{\mathbf{E}}(\mathbf{r}, \omega)=i \sqrt{\frac{\hbar}{\pi \varepsilon_{0}}} \frac{\omega^{2}}{c^{2}} \int \mathrm{d}^{3} s \sqrt{\varepsilon^{\prime \prime}(\mathbf{s}, \omega)} \mathfrak{G}(\mathbf{r}, \mathbf{s}, \omega) \cdot \hat{\mathbf{f}}(\mathbf{s}, \omega),
$$

where $\hat{\mathbf{f}}(\mathbf{s}, \omega)$ and $\hat{\mathbf{f}}^{\dagger}(\mathbf{s}, \omega)$ are creation and annihilation operators for medium-dressed states, which account for the various modes provided by the environment, such as the LSWs, surface exciton polariton and radiative modes considered in this paper.

An excited quantum emitter interacts with its environment through the electromagnetic field and relaxes from its excited state to the ground state by emitting a photon or exciting any of the dressed states supported by its environment. The initial state of the system is denoted as $|i\rangle=|e\rangle \otimes|0\rangle$, where the QE is in the excited state and the electromagnetic field is in its vacuum state. The quantum emitter will not stay indefinitely excited but will relax to the medium dressed states, and therefore the EM field will be in a $|1(\mathbf{k}, p)\rangle=\hat{f}_{i}^{\dagger}(\mathbf{r}, \omega)|0\rangle$ state; $p$ and $\mathbf{k}$ are the polarization and wave vector, respectively. The final state of the entire system therefore has the form $|f\rangle=|g\rangle \otimes \hat{f}_{i}^{\dagger}(\mathbf{r}, \omega)|0\rangle$. By applying Fermi's golden rule and summing over all final states, the expression for the $\mathrm{SE}$ rate $\Gamma$ is obtained as:

$$
\Gamma(\mathbf{r}, \omega)=\frac{2 \omega^{2} \mu^{2}}{\hbar \varepsilon_{0} c^{2}} \hat{\mathbf{n}} \cdot \operatorname{Im} \mathfrak{G}(\mathbf{r}, \mathbf{r}, \omega) \cdot \hat{\mathbf{n}},
$$

where $\hat{\mathbf{n}}$ is a unit vector along the direction of the transition dipole moment $\boldsymbol{\mu}$, and $\mathfrak{G}(\mathbf{r}, \mathbf{s}, \omega)$ is the Green's tensor representing the response of the geometry under consideration to a pointlike excitation. In order to quantify the influence of the environment on the QE emission, the normalized SE rate is defined as:

$$
\tilde{\Gamma}=\frac{\Gamma}{\Gamma_{0}}=\sqrt{\varepsilon}+\frac{6 \pi c}{\omega} \hat{n}_{i} \operatorname{Im} \mathfrak{G}_{\mathrm{S}}^{i i}(\mathbf{r}, \mathbf{r}, \omega) \hat{n}_{i},
$$

where $\varepsilon$ is the permittivity of the host medium, $\Gamma_{0}$ is given by the Einstein $A$-coefficient $\Gamma_{0}=\omega^{3} \mu^{2} / 3 \pi c^{3} \hbar \varepsilon_{0}$, and $\mathfrak{G}_{\mathrm{S}}$ is the scattering part of the Green's tensor calculated at the $\mathrm{QE}$ position $\mathbf{r}$.

The normalized SE rate for the $x$ and $z$ orientations of the transition dipole moment of a $\mathrm{QE}$ in the presence of an infinite $\mathrm{MoS}_{2}$ layer are given by the expressions

$$
\begin{aligned}
& \tilde{\Gamma}_{z}=\sqrt{\varepsilon_{1}}+\frac{3 c}{2 \omega} \operatorname{Im}\left(i \int_{0}^{\infty} \mathrm{d} k_{s} \frac{k_{s}^{3}}{k_{z 1} k_{1}^{2}} R_{N}^{11} e^{2 i k_{z 1} z}\right), \\
& \tilde{\Gamma}_{x}=\sqrt{\varepsilon_{1}}+\frac{3 c}{4 \omega} \operatorname{Im}\left[i \int_{0}^{\infty} \mathrm{d} k_{s} \frac{k_{s}}{k_{1}}\left(R_{M}^{11}+\frac{k_{z 1}^{2}}{k_{1}^{2}} R_{N}^{11}\right) e^{2 i k_{z 1} z}\right] .
\end{aligned}
$$


More details on the calculation of the Green's tensor, when an infinite $\mathrm{MoS}_{2}$ layer and superlattice are considered as the environment of a QE, are given in Appendix.

Here $R_{N}$ and $R_{M}$ are Fresnel coefficients for the reflection from the surface, defined in Appendix. For a single freestanding layer $\left(\varepsilon_{1}=\varepsilon_{2}=1\right)$, with surface conductivity $\sigma$, they are $[35,36]$

$$
\begin{array}{ll}
R_{M}^{11}=\frac{-\alpha k_{0}}{k_{z}+\alpha k_{0}}, & R_{N}^{11}=\frac{\alpha k_{z}}{k_{0}+\alpha k_{z}} \\
R_{M}^{21}=\frac{k_{z}}{k_{z}+\alpha k_{0}}, & R_{N}^{21}=\frac{k_{0}}{k_{0}+\alpha k_{z}}
\end{array}
$$

where $\alpha=2 \pi \sigma / c$ and $k_{z}=\sqrt{k_{0}^{2}-k_{s}^{2}}$.

\section{B. Surface conductivity}

$\mathrm{MoS}_{2}$ is a direct gap semiconductor with relatively intense photoluminescence [22]. The resonance part of the twodimensional optical conductivity of the $\mathrm{MoS}_{2}, \sigma_{\text {res}}$, takes into account the interaction of light with the lowest energy $A$ and $B$ excitons and is given by

$$
\sigma_{\mathrm{res}}(\omega)=\frac{4 \alpha_{0} \hbar c v^{2}}{\pi a_{\mathrm{ex}}^{2} \omega} \sum_{k=A, B} \frac{-i}{E_{k}-\hbar \omega-i \hbar \gamma_{k}},
$$

where $\alpha_{0}$ is the fine structure constant, $a_{\mathrm{ex}}=0.8 \mathrm{~nm}$ is the exciton Bohr radius, the damping parameters are $\gamma_{A}$ and $\gamma_{B}$, and the exciton energies are $E_{A}=1.9 \mathrm{eV}$ and $E_{B}=$ $2.1 \mathrm{eV} . v$ is a constant velocity, which is connected with the hopping parameter, and for $\mathrm{MoS}_{2}$ we use the value $v=0.55 \mathrm{~nm} / \mathrm{fs}[17,32]$. This optical conductivity includes only the contribution from the bright direct excitons, which dominate due to their large oscillator strength. Neither the dark nor the valley-indirect excitons appear, as they have a negligible oscillator strength. They are thus not relevant for the formation of polaritons or energy transfer to the layer, notwithstanding their important role in luminescence [37].

In Fig. 1 we present the real and imaginary parts of the surface conductivity for different values of the damping parameters, $\gamma_{A}$ and $\gamma_{B}$ [24]. The damping parameters, $\gamma_{A}$ and $\gamma_{\mathrm{B}}$, are connected with the quality of the $\mathrm{MoS}_{2}$ layer at different temperatures, and for that reason we choose to investigate a broader spectrum of parameters to account for the different mechanism of losses [38]. The real part of the surface conductivity, $\sigma_{\text {res }}(\omega)$, is connected with the losses; the higher its values, the more lossy the material. We observe in Fig. 1 that as the values of the damping parameters increase, the peaks of the real part of the surface conductivity in Fig. 1 become broader. At the exciton energies, $E_{A}$ and $E_{B}$, the losses are largest for the smallest value of the damping parameters, $\gamma_{A}$ and $\gamma_{B}$, because they give the linewidth of the resonance, but away from them the real part of $\sigma_{\text {res }}$ increases as the damping increases. The sign of the imaginary part of the surface conductivity, $\sigma_{\text {res }}(\omega)$, determines the type of modes supported by the $\mathrm{MoS}_{2}$ layer and how dispersive they are. More details on this will be given in the next section.

At even higher energies, the interband transitions need to be included in the model describing the surface conductivity.

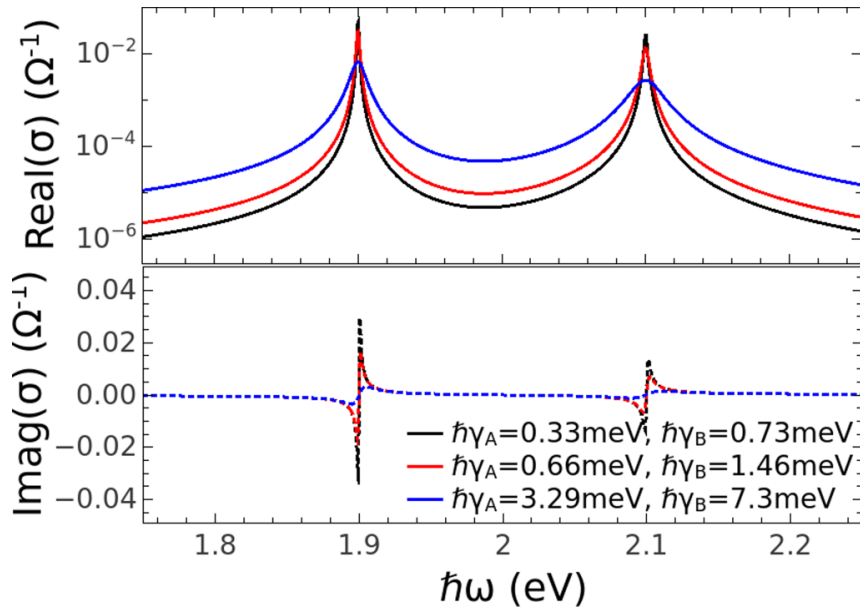

FIG. 1. Real and imaginary parts of the surface conductivity of $\mathrm{MoS}_{2}, \sigma_{\text {res }}$, given by Eq. (7) for different values of the damping parameters, $\hbar \gamma_{A}=0.3 \mathrm{meV}, 0.7 \mathrm{meV}$, and $3.3 \mathrm{meV}$ and $\hbar \gamma_{B}=$ $0.7 \mathrm{meV}, 1.4 \mathrm{meV}$, and $7.3 \mathrm{meV}$.

We model these transitions with an expression of the form

$$
\begin{aligned}
\operatorname{Real}\left(\sigma_{\text {inter }}\right)= & \frac{m \sigma_{0} \theta\left(\omega-\omega_{B}\right)}{\sqrt{1+2 E_{B} \beta+\Omega^{2}}}\left[1+\frac{1+2 E_{B} \beta}{\Omega^{2}}\left(1+E_{B} \beta\right.\right. \\
& \left.\left.-\sqrt{1+2 E_{B} \beta+\Omega^{2}}\right)\right]
\end{aligned}
$$

where $\hbar \omega_{B}=E_{B}, \Omega=\hbar \omega / E_{B}$ and $\beta$ is a mixing parameter, for $\mathrm{MoS}_{2} E_{B} \beta=0.84$ [39]. The parameter $m$ is for scaling the absorption described by Eq. (8). As we will see in Sec. III A, the effects described by Eq. (8) are not important in the energy spectrum we focus our analysis on, in particular for energies close to the exciton resonances $E_{A}$ and $E_{B}, 1.7 \mathrm{eV}<\hbar \omega<$ $2.2 \mathrm{eV}$.

The emphasis of this paper is to theoretically investigate light-matter interactions, thus, we choose to use a theoretical expression to describe the optical response of the $\mathrm{MoS}_{2}$. This is done in order to keep the discussion as general as possible. We choose the material parameters connected with $\mathrm{MoS}_{2}$, and these can be easily modified to study the interaction between a $\mathrm{QE}$ and any TMD superlattice or thin semiconducting quantum well. Furthermore, the material parameters for the $\mathrm{MoS}_{2}$ are connected with the quality of a specific sample, and thus experimentally, they vary from study to study.

\section{RESULTS}

\section{A. Surface exciton polariton modes}

We start our analysis by considering a single exciton polariton mode, shown in Fig. 2, supported by a two-dimensional material. The exciton energy is $E_{B}=2.1 \mathrm{eV}$, and the damping parameter has a value of $\hbar \gamma_{B}=0.7 \mathrm{meV}$. We use exactly the same parameters as when describing the $\mathrm{MoS}_{2}$ layer, only ignoring the exciton with energy $E_{A}$. In Fig. 2 the band structure of the transverse electric (TE) and transverse magnetic (TM) exciton polariton modes for a free standing $\left(\varepsilon_{1}=\varepsilon_{2}=1\right)$ single exciton layer are shown. The dispersion relation of the TE exciton polariton mode is calculated by 


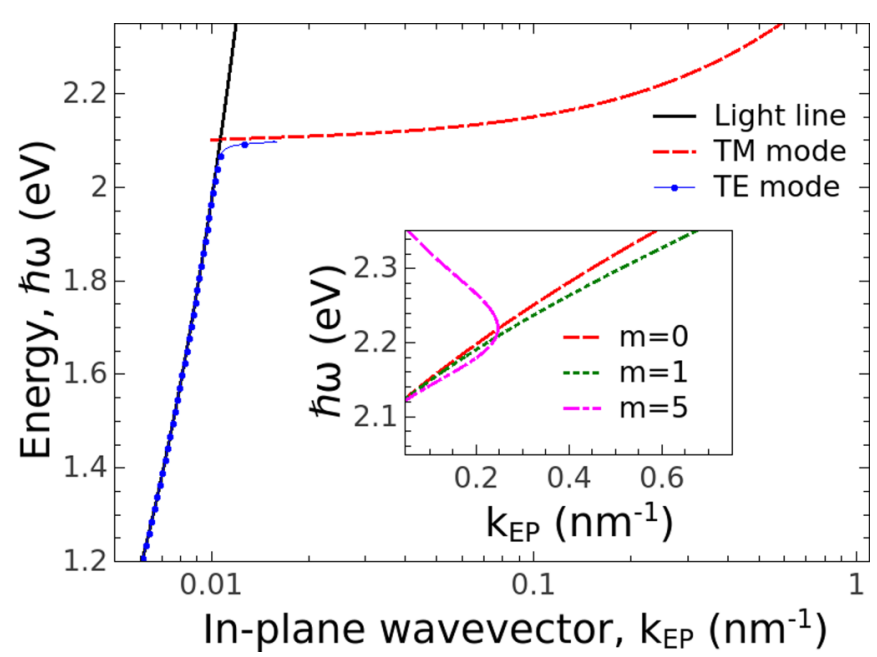

FIG. 2. Dispersion relation, $\operatorname{Re}\left(k_{\mathrm{EP}}(\omega)\right)$, for a $2 \mathrm{D}$ free-standing material, when a single exciton is considered, $E_{B}=2.1 \mathrm{eV}$. The value of the damping parameter is $\gamma_{B}=0.7 \mathrm{meV}$. In the inset the dispersion relation is presented when the interband transitions are considered, Eq. (8).

setting the denominator of $R_{M}^{11}$, Eq. (6a), to zero which gives

$$
k_{\mathrm{EP}}^{\mathrm{TE}}=\frac{\omega}{c} \sqrt{1-4 \pi^{2} \sigma_{\mathrm{res}}^{2} / c^{2}} .
$$

The TE mode is supported by the $\mathrm{MoS}_{2}$ layer only when $\operatorname{Im}\left(\sigma_{\text {res }}\right)<0$, whereas for $\operatorname{Im}\left(\sigma_{\text {res }}\right)>0$ the TE mode is on the improper Riemann sheet [40]. $k_{\mathrm{EP}}^{\mathrm{TE}}$ is the in-plane wave vector of the TE exciton polariton mode propagating on the $\mathrm{MoS}_{2}$ layer. The available TM modes are found similarly by setting the denominator of the reflection coefficient $R_{N}^{11}$, Eq. (6a), to zero, and we get the expression

$$
k_{\mathrm{EP}}^{\mathrm{TM}}=\frac{\omega}{c} \sqrt{1-c^{2} / 4 \pi^{2} \sigma_{\mathrm{res}}^{2}},
$$

which gives the dispersion relation $k_{\mathrm{EP}}^{\mathrm{TM}}(\omega)$, the relation between the frequency $\omega$, and the TM exciton polariton mode in-plane wave vector $k_{\mathrm{EP}}^{\mathrm{TM}}$. The TM exciton polariton modes can propagate on the $\operatorname{MoS}_{2}$ layer only when $\operatorname{Im}\left(\sigma_{\text {res }}\right)>0$, whereas when $\operatorname{Im}\left(\sigma_{\text {res }}\right)<0$ the TM mode given by Eq. (10) is on the improper Riemann sheet $[41,42]$.

For the case of a single exciton, the imaginary part of the surface conductivity is negative for energies below the exciton energy, $\hbar \omega<2.1 \mathrm{eV}$, thus allowing only TE exciton polariton modes to propagate. On the other hand for $\hbar \omega>2.1 \mathrm{eV}$, above the exciton energy $E_{B}$, only TM exciton polariton modes are supported. As we observe in Fig. 2, the TE modes are very close to the light line, which means that these modes are loosely confined to the $\mathrm{MoS}_{2}$ layer. It is only very close to the exciton energy $E_{B}$ that they start to become dispersive. The TM modes are clearly more dispersive, and they are tightly confined to the $\mathrm{MoS}_{2}$ layer.

In the inset of Fig. 2 the TM exciton polariton mode is presented for energies $\hbar \omega>E_{B}$, in the case when interband transitions are also included, Eq. (8). We consider the case of $m=0,1$, and 5 in Eq. (8). We observe that as the value of $m$ is increased, the dispersion relation, $\operatorname{Re}\left(k_{\mathrm{EP}}^{\mathrm{TM}}(\omega)\right)$, starts to bend back, towards the light line, for high energies, $\hbar \omega \gtrsim 2.2 \mathrm{eV}$. This is due to the higher losses caused by electron-hole pair generation. The dispersion relation has similar behavior to noble metal thin films at higher energies [43]. We observe that at energies up to $2.2 \mathrm{eV}$, the dispersion lines, for the different values of $m$, are very close. For that reason, in the rest of this paper we ignore the effect of the interband transitions, which are small in the energy range we investigate, $1.7 \mathrm{eV}<\hbar \omega<2.2 \mathrm{eV}$.

We turn the discussion now to the case in which we consider both excitons in the surface conductivity $\sigma_{\text {res }}$, which matches the physical material parameters of $\mathrm{MoS}_{2}$, Eq. (7). In Fig. 3(a) we present a plot of the dispersion relation, $\operatorname{Re}\left(k_{\mathrm{EP}}(\omega)\right)$, for the TE and TM exciton polariton modes. Due to the presence of two excitons with energies $E_{A}=1.9 \mathrm{eV}$ and $E_{B}=2.1 \mathrm{eV}$, the TE and TM exciton polariton modes split into two branches [44]. Again, an analogy can be drawn with the case of a metallic thin film, which is sandwiched between two materials with different dielectric permittivities [43]. Two surface plasmon polariton modes are present in this case, due to the two different metal-dielectric interfaces. In our case, the presence of the two excitons, with close energies, is the reason for the dispersion relation in Fig. 3. It implies that there is a change of sign for $\operatorname{Im}\left(\sigma_{\text {res }}\right)$, Fig. 1. In particular, for energies $\hbar \omega<E_{A}$ the imaginary part of the surface conductivity is negative $\operatorname{Im}\left(\sigma_{\text {res }}\right)<0$, thus only TE exciton polariton modes are supported, Fig. 3(a). These modes lie very close to the light line and are only loosely confined to the $\mathrm{MoS}_{2}$ layer, and only very close to the exciton energy $E_{A}$ do they become more dispersive. At energies $E_{A}<\hbar \omega<2 \mathrm{eV}$ the $\operatorname{Im}\left(\sigma_{\text {res }}\right)>0$, thus TM exciton polariton modes are supported which are highly dispersive, and the value of the in-plane wave vector $k_{\mathrm{EP}}^{\mathrm{TM}}$ is larger by up to two orders of magnitude than the free-space wave vector, $k_{0}=\omega / c$. At the energy of $\hbar \omega=2 \mathrm{eV}, \operatorname{Im}\left(\sigma_{\text {res }}\right) \approx 0$, and at this point the imaginary part of the surface conductivity now changes sign from plus to minus, due to the interaction between the two exciton resonances; thus, for energies $2 \mathrm{eV}<\hbar \omega<E_{B}$, we have $\operatorname{Im}\left(\sigma_{\text {res }}\right)<0$, and TE exciton polariton modes are supported. For $\hbar \omega>E_{B}, \operatorname{Im}\left(\sigma_{\text {res }}\right)>0$ and TM exciton polariton modes are again supported. In Fig. 3(a) we consider as damping parameters the values $\hbar \gamma_{A}=0.33 \mathrm{meV}$ and $\hbar \gamma_{B}=0.70 \mathrm{meV}$, and increasing these values shows small influence on the real part of the in-plane wave vector of the exciton polariton mode $k_{\mathrm{EP}} \cdot \lambda_{\mathrm{EP}}=2 \pi / k_{\mathrm{EP}}$ gives the propagation wavelength of the exciton polariton mode.

The imaginary part of the in-plane wave vector $k_{\mathrm{EP}}$ is connected with the propagation length of the exciton polariton mode $L_{\mathrm{EP}}=1 / \operatorname{Im}\left(k_{\mathrm{EP}}\right)$. In Fig. 3(b) the propagation length $L_{\mathrm{EP}}$ is shown as a function of energy for different values of the damping parameters, $\gamma_{A}$ and $\gamma_{B}$ [see the legend of Fig. 3(b) for more details]. We again observe the different intervals where the TE or TM exciton polariton modes are excited, depending on the $\operatorname{sign}$ of $\operatorname{Im}\left(\sigma_{\text {res }}\right)$. As we have already pointed out, the TE modes are loosely confined to the $\mathrm{MoS}_{2}$ layer, thus their propagation length is very large, and its value differs from the TM exciton polariton modes propagation length by up to six orders of magnitude. As the damping is increased, the propagation length $L_{\mathrm{EP}}$ decreases. It is also seen that the real part of the surface conductivity $\sigma_{\text {res }}$, which is connected with the material losses, increases at the energies of the exciton resonances with increasing damping, Fig. 1. 


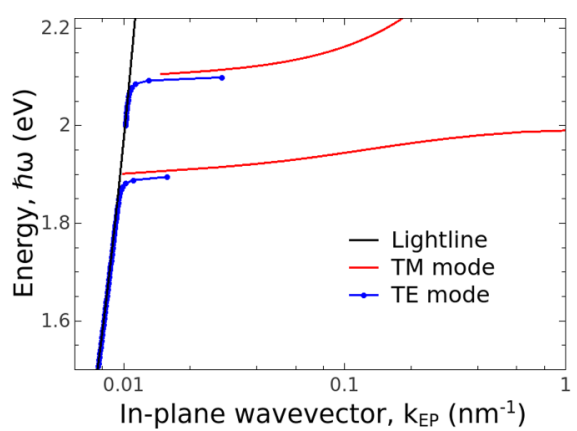

(a)

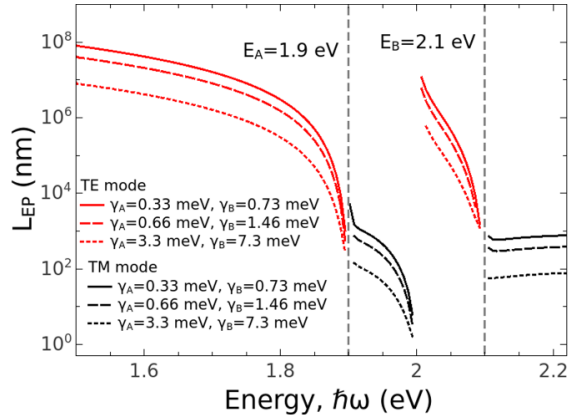

(b)

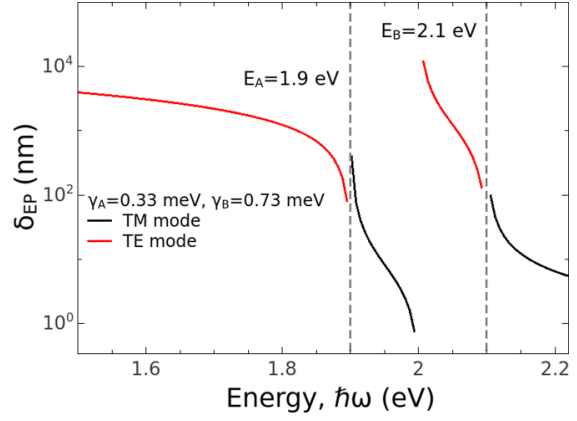

(c)

FIG. 3. (a) Plot of the dispersion relation, $\operatorname{Re}\left(k_{\mathrm{EP}}(\omega)\right.$ ), when a free standing $\operatorname{MoS}_{2}$ monolayer, $\varepsilon_{1}=\varepsilon_{2}=1$, is considered. (b), (c) Plot of the propagation length $L_{\mathrm{EP}}$ and the penetration depth $\delta_{\mathrm{EP}}$, respectively. Different values of the damping parameters, $\gamma_{A}$ and $\gamma_{B}$, are considered. More details in the legends.

The penetration depth is defined as $\delta_{\mathrm{EP}}=1 / \operatorname{Im}\left(k_{z}^{\mathrm{EP}}\right)$, where $k_{z}^{\mathrm{EP}}=\sqrt{k_{0}^{2}-k_{\mathrm{EP}}^{2}}$, and is connected with the extent of the exciton polariton mode in the direction perpendicular to the $\mathrm{MoS}_{2}$ layer. In Fig. 3(c) the penetration depth is presented as a function of energy with damping parameters $\hbar \gamma_{A}=0.33 \mathrm{meV}$ and $\hbar \gamma_{B}=0.70 \mathrm{meV}$. Again, the sign of $\operatorname{Im}\left(\sigma_{\text {res }}\right)$ gives the different intervals where the TE and TM exciton polariton modes propagate. The TE modes, only loosely confined to the $\mathrm{MoS}_{2}$ layer, are essentially radiative modes, as we will see in the next section, and have only a small contribution to the modification of the emission properties of a $\mathrm{QE}$ in proximity to the $\mathrm{MoS}_{2}$ layer.

\section{B. Spontaneous emission in the presence of a single $\mathbf{M o S}_{\mathbf{2}}$ layer}

In this section we will investigate the interaction between a QE and a MoS 2 layer. In Fig. 4 we investigate the spontaneous emission rate when we consider a $\mathrm{QE}$ at a fixed position above a free-standing $\mathrm{MoS}_{2}$ layer, as a function of its emission energy, and both $x$ and $z$ orientations for the transition dipole moment of the QE are considered in Figs. 4(a)-4(d), respectively. The total spontaneous emission is analyzed over the lossy surface wave, exciton polariton mode and radiative emission contributions. The damping parameters have the values $\hbar \gamma_{A}=0.33 \mathrm{meV}$ and $\hbar \gamma_{B}=0.70 \mathrm{meV}$.

In Fig. 4(a) the $\mathrm{QE}$ is positioned at $\mathbf{r}_{\mathrm{QE}}=(0,0,2 \mathrm{~nm})$. Due to the orientation of the transition dipole moment along $x$, TE and TM exciton polariton modes are supported by a $\mathrm{MoS}_{2}$ layer, depending on the sign of the $\operatorname{Im}\left(\sigma_{\text {res }}\right)$, as we have already discussed in Sec. II B. The contributions of these modes are obtained by extracting the pole contributions from Eq. (5b), which for $\varepsilon_{1}=\varepsilon_{2}=1$, and have the form

$$
\begin{aligned}
\tilde{\Gamma}_{x, \mathrm{EP}}\left(\omega, \mathbf{r}_{\mathrm{QE}}\right)= & \frac{3 \pi c}{4 \omega} \operatorname{Im}\left[\frac{\alpha^{2} k_{0}^{2}}{k_{z 1}^{\mathrm{TE}}} \mathrm{e}^{2 i k_{z 1}^{\mathrm{TE}} z_{\mathrm{QE}}} \Theta\left(-\operatorname{Im}\left(\sigma_{\text {res }}\right)\right)\right. \\
& \left.-\frac{k_{z 1}^{\mathrm{TM}}}{\alpha^{2}} \mathrm{e}^{2 i k_{z 1}^{\mathrm{TM}} z_{\mathrm{QE}}} \Theta\left(\operatorname{Im}\left(\sigma_{\text {res }}\right)\right)\right],
\end{aligned}
$$

where $k_{z 1}^{i}=\sqrt{k_{0}^{2}-\left(k_{\mathrm{EP}}^{i}\right)^{2}}$, for $i=\mathrm{TE}, \mathrm{TM}$ and where $k_{\mathrm{EP}}^{i}$ are given by Eqs. (9) and (10), respectively. The LSW contribution is obtained in the large $k_{s}$ limit of the integrand of Eq. (5b) [45] and has the form

$$
\begin{aligned}
\tilde{\Gamma}_{x, \mathrm{LSW}}\left(\omega, \mathbf{r}_{\mathrm{QE}}\right)= & \frac{3 c}{4 \omega} \operatorname{Im}\left[\int _ { K } ^ { \infty } \mathrm { d } k _ { s } \left(\frac{-\alpha k_{0}}{i k_{s}+\alpha k_{0}}\right.\right. \\
& \left.\left.+\frac{1}{k_{0}^{2}} \frac{i \alpha k_{s}^{3}}{k_{0}+i \alpha k_{s}}\right) \mathrm{e}^{-2 k_{s} z_{\mathrm{QE}}}\right],
\end{aligned}
$$

where the lower limit on the integral is used for numerical reasons to separate the various contributions to the full integral. In particular, when there are no TM exciton polariton modes and the TE exciton polariton modes lie very close to the light line, $K \simeq k_{0}$. When the TM modes are present, the lower integration limit should be $K>\operatorname{Re}\left(k_{\mathrm{EP}}^{\mathrm{TM}}\right)$, in order not to include the pole contribution, given by Eq. (11). The LSWs are nonpropagating dissipative modes. The radiative contribution is given by integrating Eq. (5b) over the interval $\left[0, k_{0}\right]$.

In Fig. 4(a), for emission energies of the QE below the first exciton energy, $\hbar \omega<E_{A}$, the QE's near field can excite LSWs, and these lossy modes are the main contribution to the total SE rate of the QE. Exciting the TE exciton polariton mode makes a small contribution to the total SE rate. As the emission energy of the QE is increased, in the interval $E_{A}<\hbar \omega<2 \mathrm{eV}$, the TM exciton polariton mode contribution dominates as the main channel of relaxation for the total SE rate, although the LSW still have a considerable contribution. At emission energies in the interval $2 \mathrm{eV}<\hbar \omega<E_{B}$, the LSW again dominate and the contribution of the TE exciton polariton modes is small. As we have already argued, the TE exciton polariton modes are loosely confined to the $\mathrm{MoS}_{2}$ layer, and thus their contribution to the normalized SE rate is small, see Eq. (11). Finally, for emission energies $\hbar \omega>E_{B}$, the TM exciton polariton mode contribution dominates and the LSW is suppressed, although its contribution is still considerable.

In Fig. 4(b) we observe that the enhancement of the total normalized SE rate of the $\mathrm{QE}$ placed at $\mathbf{r}_{\mathrm{QE}}=(0,0,10 \mathrm{~nm})$ is smaller when compared with the case presented in Fig. 4(a). This is due to the fact that the near field of the QE decouples from the $\mathrm{MoS}_{2}$ layer as the $\mathrm{QE}-\mathrm{MoS}_{2}$ layer distance is increased. Thus, the LSW contribution to the total SE rate along the whole spectrum is small. The LSWs can only be excited at small QE-MoS 2 separations. The TE modes also have a small contribution to the total SE rate, but they can now compete with the LSWs. However, the TM exciton polariton 


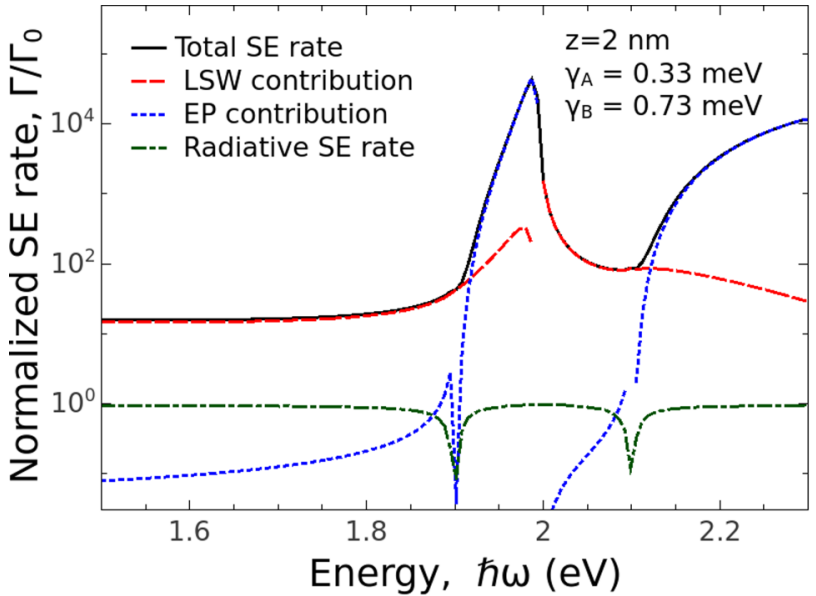

(a)

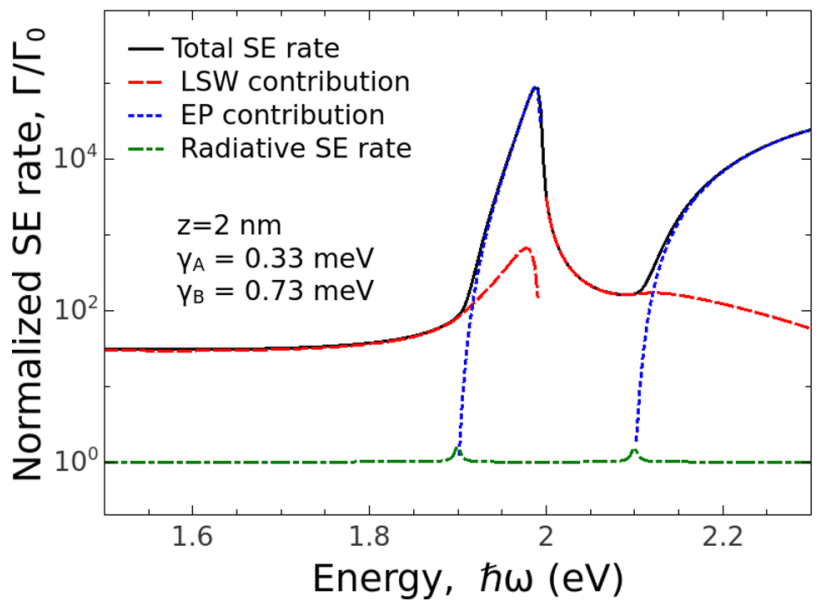

(c)

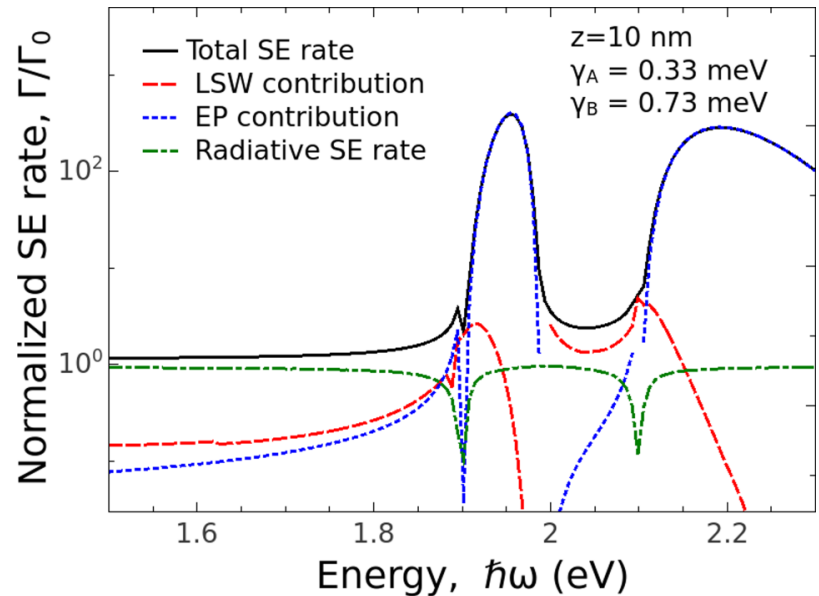

(b)

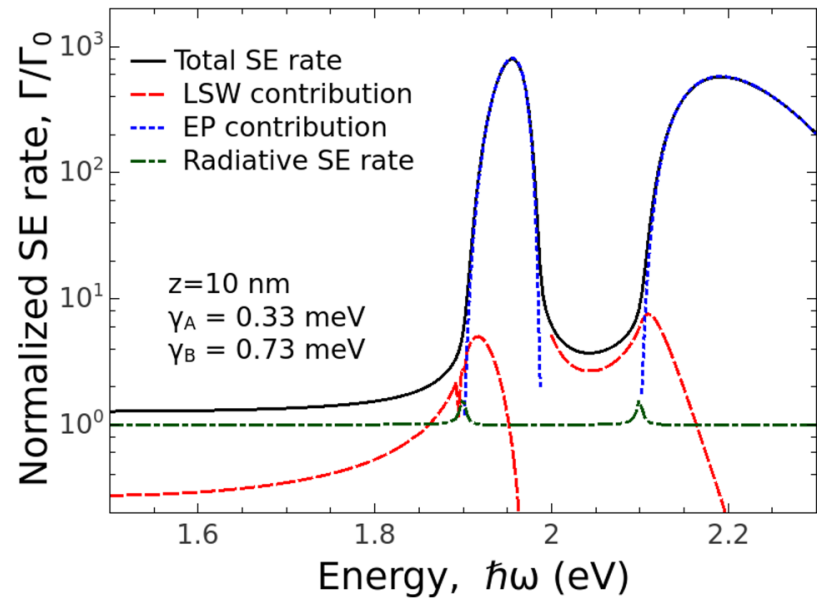

(d)

FIG. 4. The total normalized spontaneous emission of a QE, $\tilde{\Gamma}$, placed at a fixed position as a function of its emission energy, is analyzed with respect to the lossy surface wave, surface mode and radiative emission contributions. The transition dipole moment of the QE is oriented along $x$ (a),(b) and $z$ (c),(d). (a),(c) $\mathbf{r}_{\mathrm{QE}}=(0,0,2 \mathrm{~nm})$. (b),(d) $\mathbf{r}_{\mathrm{QE}}=(0,0,10 \mathrm{~nm})$. The values of the damping parameters considered are $\gamma_{A}=0.3 \mathrm{meV}$ and $\gamma_{B}=0.7 \mathrm{meV}$.

modes, in the interval where they are excited, dominate the total SE rate of the QE. The SE rate is enhanced several orders of magnitude in those intervals, compared with the free-space value.

When the transition dipole moment of the $\mathrm{QE}$ is along $z$, the pole contribution to Eq. (5a) comes exclusively from the TM exciton polariton mode and has the form

$$
\tilde{\Gamma}_{z, \mathrm{EP}}\left(\omega, \mathbf{r}_{\mathrm{QE}}\right)=-\frac{3 \pi c^{2}}{2 \omega^{2}} \operatorname{Im}\left[\frac{\left(k_{\mathrm{EP}}^{\mathrm{TM}}\right)^{2}}{\alpha} \mathrm{e}^{2 i k_{z 1}^{\mathrm{TM}} z_{\mathrm{QE}}} \Theta\left(\operatorname{Im}\left(\sigma_{\mathrm{res}}\right)\right)\right],
$$

where $k_{z 1}^{\mathrm{TM}}=\sqrt{k_{0}^{2}-\left(k_{\mathrm{EP}}^{\mathrm{TM}}\right)^{2}}$. The LSW contribution, obtained in the limit $k_{s} \rightarrow \infty$ of Eq. (5a), has the form

$$
\tilde{\Gamma}_{z, \mathrm{LSW}}\left(\omega, \mathbf{r}_{\mathrm{QE}}\right)=\frac{3 c}{2 \omega k_{0}^{2}} \operatorname{Im}\left[\int_{K}^{\infty} \mathrm{d} k_{s} \frac{i \alpha k_{s}^{3}}{k_{0}+i \alpha k_{s}} \mathrm{e}^{-2 k_{s} z \mathrm{QE}}\right],
$$

where the lower limit is determined by the existence of a TM exciton polariton mode, $K>k_{\mathrm{EP}}^{\mathrm{TM}}$ when present, and by $K \geqslant k_{0}$ when absent. The radiative contribution,
$\tilde{\Gamma}_{z, 0}\left(\omega, \mathbf{r}_{\mathrm{QE}}\right)$, is given by integrating Eq. (5a) over the interval $\left[0, k_{0}\right]$.

In Fig. 4(c) we investigate the case for which the transition dipole moment of the $\mathrm{QE}$ is along $z$, at $\mathbf{r}_{\mathrm{QE}}=(0,0,2 \mathrm{~nm})$, showing the SE rate as a function of the $\mathrm{QE}$ emission energy. Due to the dipole orientation, only TM exciton polariton modes are excited in the intervals $E_{A}<\hbar \omega<2 \mathrm{eV}$ and $\hbar \omega>E_{B}$, and these are the main channels of relaxation of the QE. The LSWs dominate the total SE rate outside the interval where TM exciton polariton modes are excited, although they also make a considerable contribution in the range $E_{A}<\hbar \omega<2 \mathrm{eV}$. In Fig. 4(d) the distance between the QE and the $\mathrm{MoS}_{2}$ layer is increased to $\mathbf{r}_{\mathrm{QE}}=(0,0,10 \mathrm{~nm})$. The LSW contribution decreases as the distance between $\mathrm{QE}$ and the $\mathrm{MoS}_{2}$ layer increases. The total SE rate is enhanced several orders of magnitude when the TM exciton polariton mode is excited. In general, the SE rate of a $\mathrm{QE}$ has similar characteristics for the $x$ and $z$ polarizations. The main difference is that, for a $\mathrm{QE}$ with a transition dipole moment along $x$, TE exciton polariton modes can be excited. The coupling between a $\mathrm{QE}$ and the 


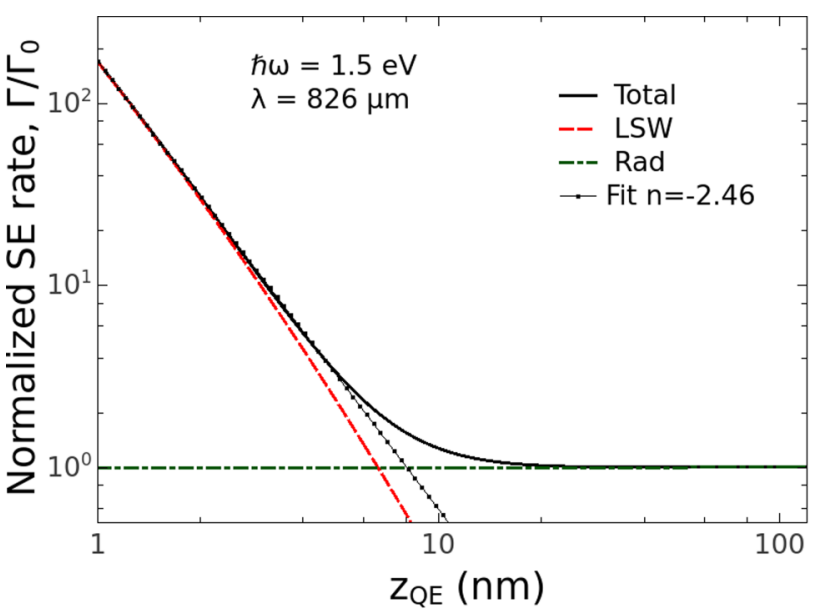

(a)

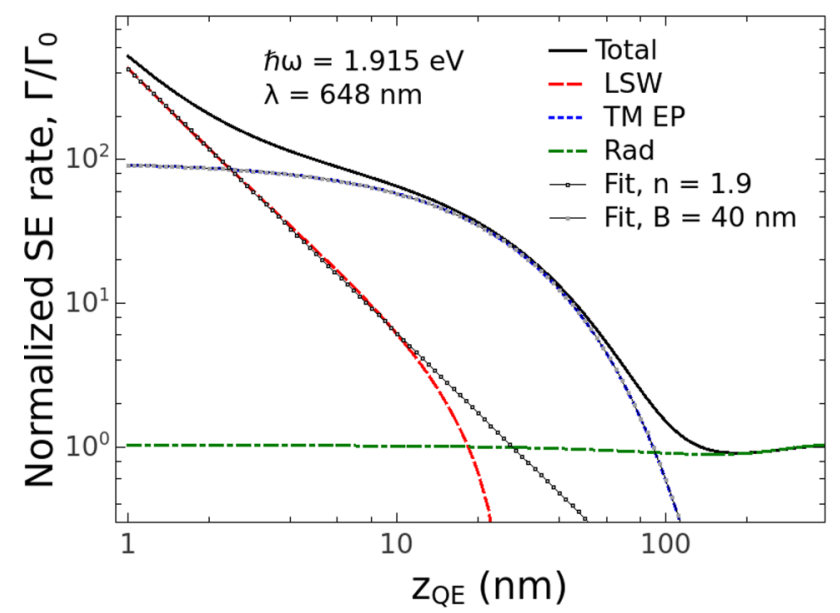

(c)

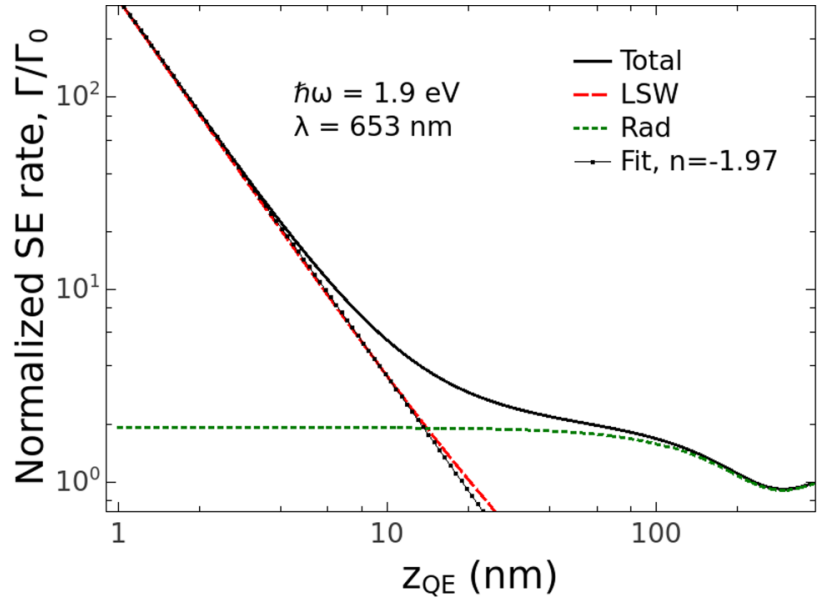

(b)

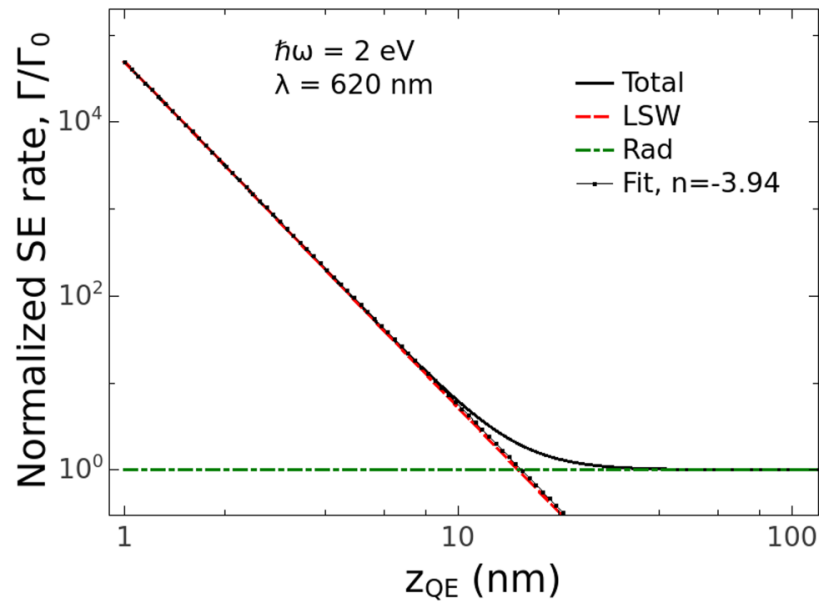

(d)

FIG. 5. The total normalized spontaneous emission rate of a $\mathrm{QE}$, with fixed emission energy as a function of its position $\mathbf{r}=\left(0,0, z_{\mathrm{QE}}\right)$, is analyzed with respect to the lossy surface wave, exciton polariton mode and radiative emission contributions. The transition dipole moment of the QE is $z$ oriented. (a) $\hbar \omega=1.5 \mathrm{eV}$. (b) $\hbar \omega=1.9 \mathrm{eV}$. (c) $\hbar \omega=1.915 \mathrm{eV}$. (d) $\hbar \omega=2.0 \mathrm{eV}$. The damping parameters have the values $\gamma_{B}=0.3 \mathrm{meV}$ and $\gamma_{A}=0.7 \mathrm{meV}$.

$\mathrm{MoS}_{2}$ layer is more efficient for the $z$ orientation. For the rest of this paper we focus on a QE with $z$ orientation of the transition dipole moment.

The distance dependence of the interaction between QEs and a TMD monolayer has been investigated experimentally, and different theoretical expressions have been used to fit the experimental results. In Ref. [31] they report a $z^{-4}$ behavior of the distance dependence of the interaction between a $\mathrm{QE}$ and a $\mathrm{MoSe}_{2}$ layer, although the authors fit the intensity quenching rather than the lifetime quenching. On the other hand, Ref. [28] uses multiple QEs and investigates their lifetime quenching in the presence of a $\mathrm{MoS}_{2}$ monolayer. The extracted fittings for the lifetime quenching are between $z^{-3}$ to $z^{-4}$.

In Fig. 5 we present the distance dependence of the spontaneous emission rate of a $\mathrm{QE}$, placed at $\mathbf{r}_{\mathrm{QE}}=\left(0,0, z_{\mathrm{QE}}\right)$, and oriented along $z$, for fixed emission energies. We analyze the different contributions to the total SE rate, the LSWs, TM exciton polariton modes and the radiative emission using Eqs. (5a), (13), and (14). We consider four values of the emission energy of the QE, one in the range $\hbar \omega<E_{A}$, where the LSWs dominate and one in the $E_{A}<\hbar \omega<2 \mathrm{eV}$ range, where the TM exciton polariton modes are excited. The other two values are at $\hbar \omega=1.9 \mathrm{eV}$, right on the exciton energy $E_{A}$, and $\hbar \omega=2.0 \mathrm{eV}$, at the position where $\operatorname{Im}\left(\sigma_{\text {res }}\right)$ changes sign due to the interaction between the two excitons.

In Fig. 5(a) the QE emission energy is $\hbar \omega=1.5 \mathrm{eV}$, in the interval $\hbar \omega<E_{A}$, thus we see that the main contribution comes from the LSWs very close to the $\mathrm{MoS}_{2}$ layer, but this channel of interaction dies out quickly and at separations as small as $z_{\mathrm{QE}} \approx 8 \mathrm{~nm}$, the $\mathrm{SE}$ rate reverts to its free-space value. At this energy there is no exciton polariton mode, due to the dipole moment orientation of the $\mathrm{QE}$. The integral in Eq. (14) has contributions of the form $A_{1} / z^{2}+A_{2} / z^{3}+$ $A_{3} / z^{4}$, therefore in order to analyze the LSW contribution, we use the fitting expression:

$$
f(z)=A z^{n}
$$

and in Fig. 5(a) we show that $n=-2.5$. This fitting shows that the behavior of the $\mathrm{MoS}_{2}$ layer is very different from the case of a graphene layer in the optical part of the spectrum. The optical response of graphene is constant and 
characterized by a surface conductivity of $\sigma_{\mathrm{Graph}}=\sigma_{0}=$ $e^{2} / 2 \hbar$. The distance dependence of the $\mathrm{SE}$ rate of a $\mathrm{QE}$ is then given by $\tilde{\Gamma} \propto 1 / z^{4}$, Eq. (14), which is a universal scaling law of the distance dependence between a QE and a graphene monolayer, in the optical part of the spectrum [8,9,30]. In Fig. 5(b) the QE energy is $\hbar \omega=E_{A}=1.9 \mathrm{eV}$, and we observe a behavior similar to Fig. 5(a), but now the fitting of the LSWs, which have the largest contribution to the total $\mathrm{SE}$ rate, gives $n \approx-2$ and the $\mathrm{QE}$ reverts to the radiative value of the $\mathrm{SE}$ at distances of $10 \mathrm{~nm}$. We furthermore observe that the radiative $\mathrm{SE}$ rate of the $\mathrm{QE}$ is enhanced very close to the $\mathrm{MoS}_{2}$, which is an effect of constructive interference with the image dipole, due to the dipole orientation.

In Fig. 5(c) the emission energy of the QE is $\hbar \omega=$ $1.915 \mathrm{eV}$, and we observe that the main contribution to the SE rate close to the $\mathrm{MoS}_{2}$ layer again comes from the LSWs. At this energy a TM exciton polariton mode is excited and thus adds a new path of relaxation for the QE. The TM exciton polariton mode has a considerable contribution at small separations between the QE and the $\mathrm{MoS}_{2}$ layer and dominates at intermediate distances, $6 \mathrm{~nm}<z_{\mathrm{QE}}<100 \mathrm{~nm}$. In order to better understand the influence of the TM modes on the SE rate, we use a fitting expression of the form:

$$
g\left(z_{\mathrm{QE}}\right)=A \exp \left(-2 z_{\mathrm{QE}} / B\right),
$$

where $B$ is the fitting parameter of interest, connected with the penetration depth of the TM exciton polariton mode, $\delta_{\mathrm{EP}}=1 / \operatorname{Im}\left(k_{z}^{\mathrm{EP}}\right)$. The value of the fitting parameter in Fig. 5(c) is found to be $B=40 \mathrm{~nm}$ which is the same as the value plotted in Fig. 3(c) where $\delta_{\mathrm{TM}}=40 \mathrm{~nm}$. The distance dependence of LSWs is described by Eq. (15) with $n \approx-2$. The LSW contribution to the SE rate is calculated using the approximate expression Eq. (14). When the TM modes are also present, it becomes more challenging to distinguish between the propagating TM exciton polariton and the nonpropagating LSW nature of the relaxation. In Fig. 5(d) the emission energy is $\hbar \omega=2 \mathrm{eV}$, and at this energy there is a change of sign of the $\operatorname{Im}\left(\sigma_{\mathrm{MoS}_{2}}\right)$ from positive to negative values while the ratio $\left.\operatorname{Re}\left(\sigma_{\text {res }}\right)\right) / \operatorname{Im}\left(\sigma_{\text {res }}\right) \gg 1$, thus we can safely ignore the imaginary part. We then have a situation identical to graphene and the LSWs, which dominate at small QE-MoS layer separations, follow a behavior given by Eq. (15) with $n \approx-4$.

For completeness we present the full spectral and distance dependence of the SE rate of a $\mathrm{QE}$ in the presence of a $\mathrm{MoS}_{2}$ layer, in Fig. 6. This is a contour plot of the normalized total SE rate of a $\mathrm{QE}$, as a function of the $\mathrm{QE}$ position $\mathbf{r}_{\mathrm{QE}}=\left(0,0, z_{\mathrm{QE}}\right)$ and its emission energy $\hbar \omega$. The transition dipole moment of the QE is along $z$. The olive green line represents the boundary of the parameter space where $\Gamma / \Gamma_{0}>10$. We observe that at emission energies where one can excite the TM exciton polariton mode supported by the $\mathrm{MoS}_{2}$ layer, at $1.9 \mathrm{eV}<$ $\hbar \omega<2 \mathrm{eV}$ and $\hbar \omega>2.1 \mathrm{eV}$, the SE rate is enhanced up to 10 times, compared with its free-space value, for distances up to $40 \mathrm{~nm}$. At small distances, the SE rate is enhanced due to the excitation of the nonpropagating LSWs. The values of the damping parameters considered are $\hbar \gamma_{A}=0.3 \mathrm{meV}$ and $\hbar \gamma_{B}=0.7 \mathrm{meV}$.

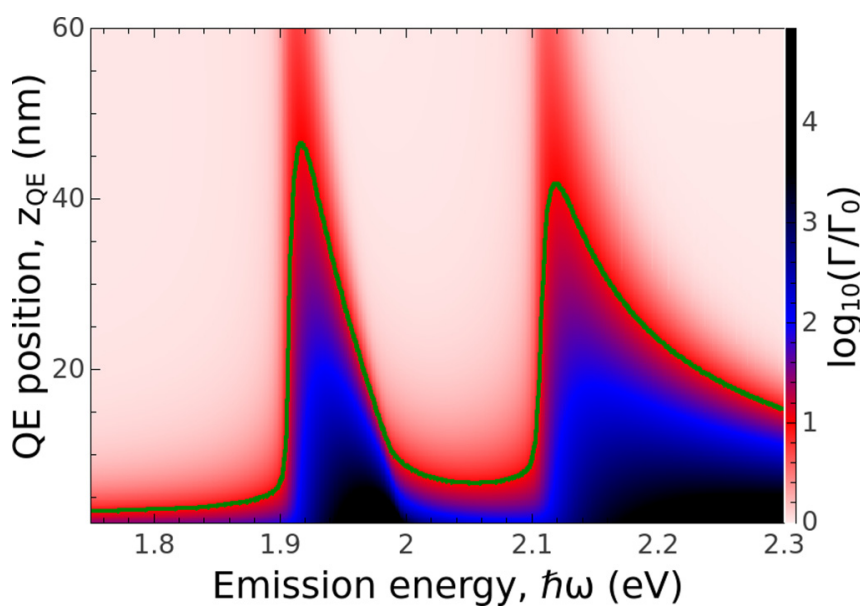

FIG. 6. Contour plot of the total normalized SE of a QE, as a function of its position, $\mathbf{r}_{\mathrm{QE}}=\left(0,0, z_{\mathrm{QE}}\right)$, and emission energy $\hbar \omega$. The transition dipole moment of the $\mathrm{QE}$ is $z$ oriented. The values of the damping parameters that are considered are $\gamma_{A}=0.3 \mathrm{meV}$ and $\gamma_{B}=0.7 \mathrm{meV}$.

\section{Spontaneous emission in the presence of a superlattice of $\mathrm{MoS}_{2}$ layers}

In this section we investigate the influence of the presence of a superlattice composed of multiple $\mathrm{MoS}_{2}$ layers on the emission properties of a QE. There are contradicting experimental reports regarding the influence on the $S E$ rate of a QE interacting with TMD layers, as the number of layers is increased. In particular, in Refs. [26,30], the authors report that, as the number of $\mathrm{MoS}_{2}$ layers is increased, the SE rate of the QEs decreases. The authors of Ref. [30] use a bulk dielectric permittivity to describe the optical response of the $\mathrm{MoS}_{2}$, and they attribute the decreasing behavior to dielectric screening [46]. In particular, they found that, by increasing the thickness of the $\mathrm{MoS}_{2}$ slab, the field intensity created by a dipole source on the slab drops. The screening effect is connected with the difference between the parallel and perpendicular dielectric permittivities of the $\mathrm{MoS}_{2}$ slab; more details can be found in Ref. [30]. Also, the real part of the dielectric permittivity has larger values compared with the imaginary part, further increasing the screening effect [46]. Their analysis is focused on a single emission energy of the QE. On the other hand, in Ref. [29], the authors report an opposite behavior where, as the number of layers of $\mathrm{SnS}_{2}$ is increased, the SE rate also increases. This discrepancy is attributed to the fact that the $\mathrm{MoS}_{2}$ material exhibits a band inversion from indirect, as a bulk material, to direct as a monolayer, while $\mathrm{SnS}_{2}$ is an indirect band gap material down to a monolayer. Furthermore, in Refs. [26,29,30], the emission profile of the QEs investigated is different for each case.

Our analysis follows a different path. Instead of using a slab for approximating the $\mathrm{MoS}_{2}$ layer, and describing its optical response through an anisotropic dielectric permittivity, we treat the $\mathrm{MoS}_{2}$ as a 2D material, whose optical response is given by Eq. (7). We describe the interaction between a $\mathrm{QE}$ and a $\mathrm{MoS}_{2}$ superlattice using Eqs. (A2) and (A3). Multiple scattering between the $\mathrm{MoS}_{2}$ layers creates a number of modes, depending on the number of layers. We analyze and investigate 


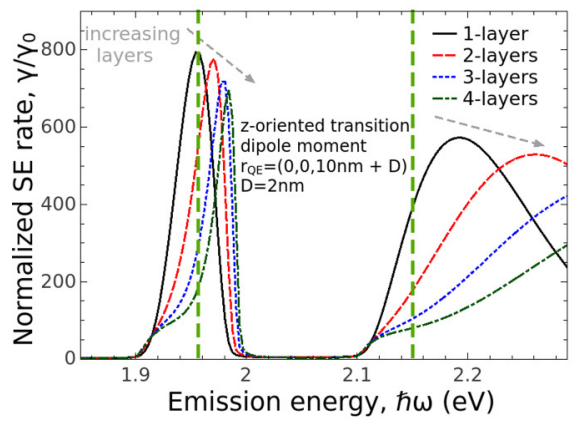

(a)

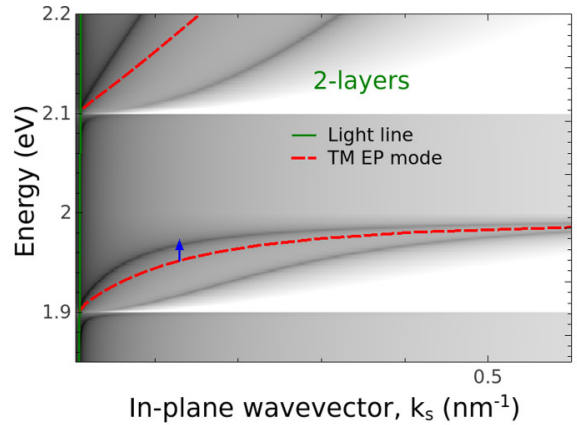

(b)

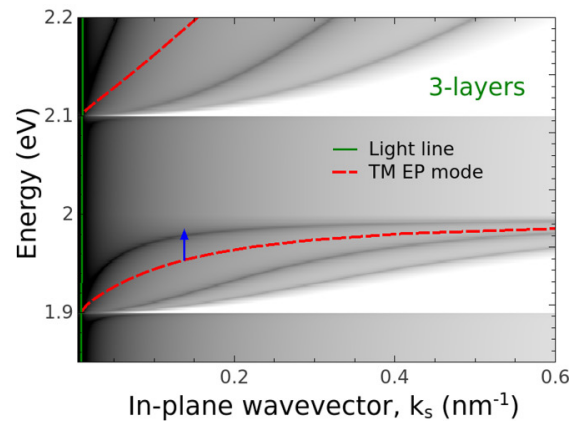

(c)

FIG. 7. (a) Total normalized spontaneous emission of a $\mathrm{QE}$, placed at a fixed position, $\mathbf{r}_{\mathrm{QE}}=(0,0,10 \mathrm{~nm}+D)$, as a function of its emission energy, considering different numbers of $\mathrm{MoS}_{2}$ layers. The transition dipole moment of the QE is $z$ oriented. The dashed vertical lines correspond to the peak emission energies of the quantum dots in Refs. [26,30]. (b),(c) Dispersion relation $k_{s}(\omega) \operatorname{showing} \log \left(\left|R_{N}\right|\right)$, for $\operatorname{Im}\left(\sigma_{\text {res }}\right)>0$, and $\log \left(\left|R_{M}\right|\right)$, for $\operatorname{Im}\left(\sigma_{\text {res }}\right)<0$, considering multiple $\mathrm{MoS}_{2}$ layers. (b) Two layers. (d) Three layers. $D$ is the thickness between the lower and upper $\mathrm{MoS}_{2}$ layers, here $D=2 \mathrm{~nm}$. The dispersion relation of the TM exciton polariton mode of a single $\mathrm{MoS}_{2}$ layer is presented with a red dashed line. The damping parameters have the values $\gamma_{B}=0.3 \mathrm{meV}$ and $\gamma_{A}=0.7 \mathrm{meV}$.

the influence these modes have on the total SE rate of the QE. We choose to investigate free standing $\mathrm{MoS}_{2}$ superlattices for simplicity. The inclusion of the substrate will slow down the SE rate due to the difference between the substrate and superlattice dielectric permittivities. The main relaxation path for a $\mathrm{QE}$ is associated with the exciton polariton modes, provided by the $\mathrm{MoS}_{2}$ superlattice. Their existence is unaffected by the inclusion of a substrate.

In Fig. 7(a) we present the $\mathrm{SE}$ rate as a function of emission energy considering the interaction between a $\mathrm{QE}$ and a monolayer and, double, triple, and quadruple $\mathrm{MoS}_{2}$ layers. Again we consider the case where the transition dipole moment of the $\mathrm{QE}$ is oriented along $z$. The position of the $\mathrm{QE}$ is fixed at $\mathbf{r}_{\mathrm{QE}}=(0,0,10 \mathrm{~nm}+D)$, where $D$ is the distance between the top and bottom layers. We consider a fixed value of this thickness, $D=2 \mathrm{~nm}$, and the distance between the layers is kept equal. So as the number of layers is increased the distance between them is decreased. In Fig. 7(a) we observe that, as the number of layers increases, the peak value of the normalized SE rate blueshifts and the absolute value of its enhancement decreases. The shift is smaller when the lower TM exciton polariton mode is excited. In Refs. [26,30] the authors experimentally found that the SE rate of CdSe/CdZnS quantum dots, with peak emissions at $1.95 \mathrm{eV}$ and $2.15 \mathrm{eV}$, decreases when interacting with an increasing number of $\mathrm{MoS}_{2}$ layers. As can be seen in Fig. 7(a), this is consistent with our observation and hence may be due to the coupling between the layers through the electromagnetic field. However, our theory does not account for the direct electronic coupling between layers, which may also play a role. In order to give an explanation for this effect we present in Figs. 7(b) and 7(c) the dispersion relation for two superlattice examples.

As we have already discussed, the TE and TM exciton polariton modes are obtained as poles of the generalized Fresnel reflection coefficients. For a superlattice nanostructure more details are given in Appendix. In Figs. 7(b) and 7(c) we present a contour plot of the logarithm of the absolute value of the reflection coefficients $R_{N}\left(k_{s}, \omega\right)$, for $E_{A}<\hbar \omega<2.0 \mathrm{eV}$ and $\hbar \omega>E_{B}$, and $R_{M}\left(k_{s}, \omega\right)$, for $\hbar \omega<E_{A}$ and $2.0 \mathrm{eV}<$ $\hbar \omega<E_{B}$, as a function of the in-plane wave vector $k_{s}$ and the energy $\hbar \omega$. The generalized reflection coefficients $R$ are calculated by solving Eq. (A4). The TM mode has the largest contribution to the SE rate, see Fig. 7(a). The dispersion relation lines are given by the dark color lines in the contour plot. We observe that as the number of layers increases, more branches emerge in the energy range where TM modes are supported by the $\mathrm{MoS}_{2}$ superlattice, and the number of branches is equal to the number of layers. These extra branches are connected with the multiple scatterings in the $\mathrm{MoS}_{2}$ superlattice. In the same figure we present with a red dashed line the dispersion relation of a single $\mathrm{MoS}_{2}$ layer for direct comparison.

The peak in the SE rate enhancement of a $\mathrm{QE}$ for a single layer is at $\hbar \omega=1.95 \mathrm{eV}$, Fig. 7(a). The main channel of relaxation of the QE, in the presence of the $\mathrm{MoS}_{2}$ superlattice, is the TM exciton polariton mode. We choose to focus on the lower branch of the TM exciton polariton mode, related to the first peak of the normalized SE rate in Fig. 7(a). The peak value for the single layer is connected with the penetration depth, $\delta_{\mathrm{EP}}^{\mathrm{TM}}=1 / \operatorname{Im}\left(k_{z}^{\mathrm{TM}}\right)$, where $k_{z}^{\mathrm{TM}}=\sqrt{k_{0}^{2}-k_{\mathrm{EP}}^{\mathrm{TM}}} \approx i k_{\mathrm{EP}}^{\mathrm{TM}}$ and since $k_{\mathrm{EP}}^{\mathrm{TM}} \gg k_{0}$, we find $\delta_{\mathrm{EP}}^{\mathrm{TM}}=1 / \operatorname{Re}\left(k_{\mathrm{EP}}^{\mathrm{TM}}\right)$. In Eq. (13), the exciton polariton contribution to the SE rate depends on a factor $c^{2} / \omega^{2}$, thus for the same value of $k_{\mathrm{EP}}^{\mathrm{TM}}$, there is a decrease in the absolute value of the normalized SE rate with increasing energy, explaining the trend we observe in Fig. 7(a). In Figs. 7(b) and 7(c), we show the position of the peak value of the SE rate of the QE interacting with a single $\mathrm{MoS}_{2}$ layer, and the blue arrow indicates the blueshift of the energy at which the peak value of the SE rate emerges in Fig. 7(a) when the double layer is considered. For the single layer, the peak of the $\mathrm{SE}$ rate is at $\hbar \omega=1.95 \mathrm{eV}$ at $k_{\mathrm{EP}}^{\mathrm{TM}}=0.1 \mathrm{~nm}^{-1}, \delta_{\mathrm{EP}}^{\mathrm{TM}}=10 \mathrm{~nm}$, while for the double layer the peak is at $\hbar \omega=1.97 \mathrm{~nm}$ and for the triple layer it is at $\hbar \omega=1.98 \mathrm{~nm}$. Thus the dispersion relations give an explanation for the blueshift of the peak value of the normalized SE rate.

Therefore, to investigate the interaction between a specific $\mathrm{QE}$ and a $\mathrm{MoS}_{2}$ superlattice, one must take into account the reduced interaction between QEs- $\mathrm{MoS}_{2}$ superlattice as one starts increasing the number of layers. While the emission 


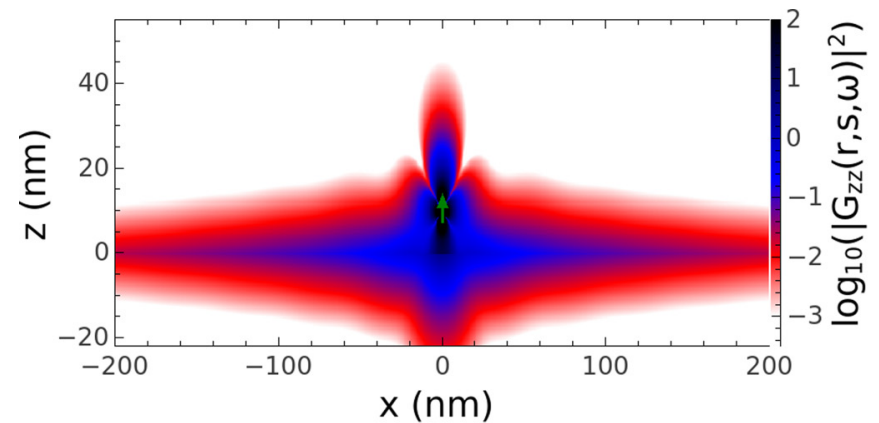

(a)

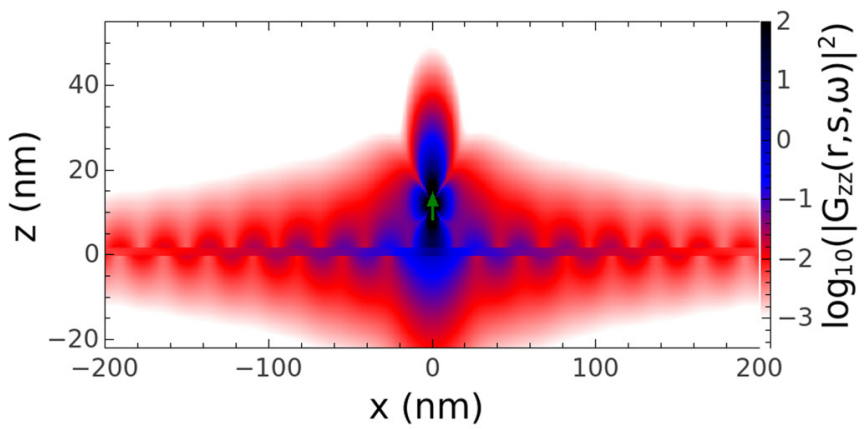

(b)

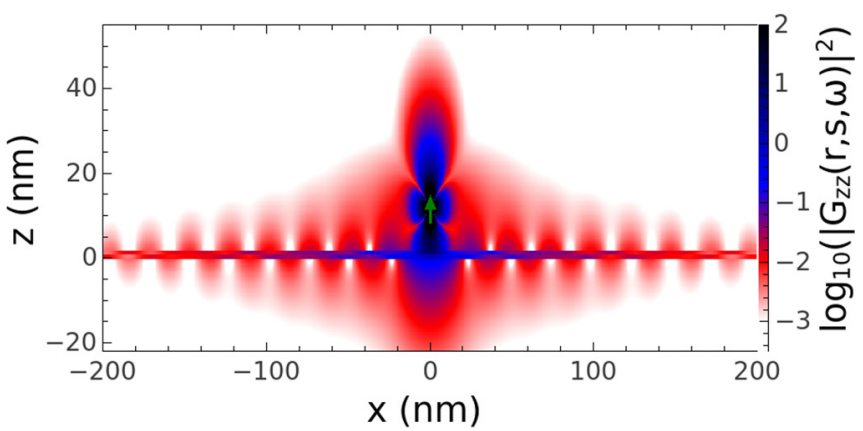

(c)

FIG. 8. Contour plots of the logarithm of the field intensity, $\log _{10}\left(\left|G_{z z}(\mathbf{r}, \mathbf{s}, \omega)\right|^{2}\right)$, created by a QE, placed at $\mathbf{s}=(0,0, D+$ $10 \mathrm{~nm})$. The transition dipole moment of the QE is along $z$, and its emission energy is $\hbar \omega=1.915 \mathrm{eV}$. (a) Single layer, (b) double layer, (c) triple layer. The damping parameters have the values $\gamma_{B}=0.3 \mathrm{meV}$ and $\gamma_{A}=0.7 \mathrm{meV}$.

properties of the QE do not change, its environment is modified as there is a redistribution of the available modes. The dispersion relation plays a crucial role in explaining this effect, giving us the available modes that can be supported.

To further analyze this effect, in Fig. 8 we present contour plots of the logarithm of the absolute value of the electric field, $E_{z}(\mathbf{r}, \omega) \propto \mathfrak{G}_{z z}\left(\mathbf{r}, \mathbf{r}_{\mathrm{QE}}, \omega\right)$, created by a $\mathrm{QE}$ placed at $\mathbf{r}_{\mathrm{QE}}=$ $(0,0,10 \mathrm{~nm}+D)$, in the presence of a $\mathrm{MoS}_{2}$ superlattice, $D=$ $2 \mathrm{~nm}$ in our case. The emission energy of the QE has been selected to be at the maximum value of the SE rate for a single $\mathrm{MoS}_{2}$ layer, $\hbar \omega=1.95 \mathrm{eV}$. The scale of the color maps in Fig. 8 is the same in all panels, for direct comparison. We observe that the field intensity decreases as the number of the $\mathrm{MoS}_{2}$ layers increases. This is due to the poorer coupling of the near field of the $\mathrm{QE}$ to the $\mathrm{MoS}_{2}$ superlattice. We observe also that the extent of the field in the $x$ direction decreases as the number of layers is increased. The propagation length for the single layer is $L_{\mathrm{EP}}^{\mathrm{TM}}=373 \mathrm{~nm}$ while for the double layer it is $L_{\mathrm{EP}}^{\mathrm{TM}}=300 \mathrm{~nm}$, and, as the number of layers further increases, the propagation length further reduces.

The opposite behavior can be observed when the QE emission energy is at the maximum value of the SE rate for a $\mathrm{MoS}_{2}$ superlattice, e.g., for the three-layer superlattice. Then the SE rate of the QE decreases with decreasing the number of layers. For this example of the trilayer, at the resonance $\hbar \omega=1.98$, the normalized SE rate is $\tilde{\Gamma}=718$, compared with $\tilde{\Gamma}=444$ for the two layer and $\tilde{\Gamma}=102$ for a monolayer.

\section{CONCLUSIONS AND FUTURE WORK}

In this contribution we have investigated the spectral and distance dependence of the SE rate of a QE in the presence of a $\mathrm{MoS}_{2}$ layer and superlattice. A $\mathrm{MoS}_{2}$ layer supports transverse electric and transverse magnetic surface exciton polariton modes. The TM modes are strongly confined to the $\mathrm{MoS}_{2}$ layer and have long propagation lengths. The TE modes are only loosely confined to the $\mathrm{MoS}_{2}$ layer.

The total SE rate of the QE in the presence of a $\mathrm{MoS}_{2}$ layer is analyzed with respect to the different contributions, namely, the lossy surface wave, surface exciton polariton and radiative emission. In the main part of the discussion we showed that the existence of TE and TM exciton polariton modes is connected with the surface conductivity of the $\mathrm{MoS}_{2}$, specifically with the sign of its imaginary part. For energies at which the TM exciton polariton modes are excited, the SE rate of a $\mathrm{QE}$ is enhanced by several orders of magnitude, compared with its free-space value. For all emission energies of the QE, the main contribution to the SE comes from the LSW at small separations, but their contribution dies out fast as the separation is increased. When the TM modes are excited, they dominate at intermediate distances, $6 \mathrm{~nm}$ to $100 \mathrm{~nm}$. For distances at which the LSWs are not excited or above the penetration depth of the TM exciton polariton modes, the QE radiates to the far field.

Next, the interaction between a $\mathrm{QE}$ and a $\mathrm{MoS}_{2}$ superlattice is investigated. We observe a blueshift of the peak value of the $\mathrm{SE}$ rate of the $\mathrm{QE}$, as a function of its emission energy, as we increase the number of $\mathrm{MoS}_{2}$ layers from one to four. Using the dispersion relation plot, this blueshift is explained. It is seen that the number of layers determines the number of branches of the exciton polariton modes available. The blueshift of the SE rate is connected with the blueshift of the dispersion line for the $\mathrm{MoS}_{2}$ superlattice, compared with the single layer. For a QE with emission energy corresponding to the peak energy of the SE rate of a monolayer, as the number of layers is increased the coupling decreases and the field intensity distribution around the superlattice decreases. The opposite behavior is observed if the emission energy of the $\mathrm{QE}$ is on resonance with a $\mathrm{MoS}_{2}$ superlattice.

Although the results presented in this study focused on $\mathrm{MoS}_{2}$ as a material, they are quite general and can be applied to any material whose optical properties are determined by exciton generation. Thus, they can be applied to any of the rest of the TMD family. Furthermore, we have chosen to 
concentrate on a theoretical investigation of the $\mathrm{MoS}_{2}$ and not to fit existing experimental data. This is due to the fact that the material parameters are strongly influenced by the quality of the material sample itself.

This study made a contribution to explaining all the contradictory results regarding the spectral and distance dependences of QEs in the presence of $\mathrm{MoS}_{2}$ layers. Specifically, we presented a $z^{n}, n=2,3,4$, distance dependence of the SE rate of a $\mathrm{QE}$, which is connected with the LSWs at the different emission energies. Also we observed the existence of exciton polariton modes and how they modify the emission properties of QEs and the strength of the interaction, where the distance dependence follows the expression $\sim \exp \left(-2 z / \delta_{E P}^{T M}\right)$. Moreover, we explained that the coupling of a QE with a $\mathrm{MoS}_{2}$ superlattice depends on its emission energy. The peak value for the SE rate of a QE blueshifts depending on the number of $\mathrm{MoS}_{2}$ layers, due to the different mode distributions supported by these structures. While the emission properties of the QE remain the same, the modes provided by the environment change as the number of $\mathrm{MoS}_{2}$ layers changes. Depending on the emission energy of the $\mathrm{QE}$, the $\mathrm{SE}$ rate can increase or decrease as the number of $\mathrm{MoS}_{2}$ layers is increased. Dielectric screening can explain certain results for certain emission energies of the QE.

Multilayer devices based on $\mathrm{MoS}_{2}$ and graphene can be the precursors of an all-optical device. Graphene's optical properties can be tuned by changing its chemical potential, but it has small absorption in the visible part of the optical spectrum. Combining graphene with TMD layers allows one access to the best of both materials for applications such as light harvesting and light emitting devices $[47,48]$. In general the total absorption of these two materials can be further enhanced by also including layers of QEs. The emission properties of the QEs can be chosen depending on the nature of the application. For light harvesting devices we need the emission energy of the QEs to maximize the nonradiative energy transfer to the $\mathrm{MoS}_{2}$ layer, where the generated electron-hole pair will be harvested. On the other hand, for LEDs we need to maximize the far field emission of the SE rate of the QEs. Both of these effects can be further investigated for devices composed from patterned 2D nanostructures, like ribbons and disks, where the redistribution of the available modes gives rise to sharp resonances $[49,50]$.

\section{ACKNOWLEDGMENT}

This work was supported by the Science Foundation Ireland under Grant No. 10/IN.1/12975.

\section{APPENDIX: GREEN'S TENSOR FOR A MoS 2 SUPERLATTICE}

A multilayer planar geometry is considered, which consists of a number of $N$ layers, indexed by their layer number $i=1, \ldots, N$ where each layer has thickness $d_{i}$ and dielectric permittivity $\varepsilon_{i}$. The layers are of infinite extent in the $x y$ plane, and the $z$ axis is perpendicular to the surface of each layer.

The method of scattering superposition is used [51,52] where the Green's tensor splits into two parts:

$$
\mathfrak{G}(\mathbf{r}, \mathbf{s}, \omega)=\mathfrak{G}_{h}(\mathbf{r}, \mathbf{s}, \omega)+\mathfrak{G}_{s}(\mathbf{r}, \mathbf{s}, \omega),
$$

where $\mathfrak{G}_{h}(\mathbf{r}, \mathbf{s}, \omega)$ is the homogeneous part that accounts for direct interaction between the source and target points at $\mathbf{s}$ and $\mathbf{r}$, respectively, and is nonzero when both points are in the same medium and there is no discontinuity between them. $\mathfrak{G}_{s}(\mathbf{r}, \mathbf{s}, \omega)$ is the scattering part, it is always present, and accounts for the multiple reflections and transmissions taking place at the interfaces.

The general form of the scattering part of the Green's tensor has the form:

$$
\begin{aligned}
\mathfrak{G}_{s}(\mathbf{r}, \mathbf{s}, \omega)= & \frac{i}{8 \pi^{2}} \int \mathrm{d}^{2} k_{s} \frac{1}{k_{z i} k_{s}^{2}} \sum_{T} R_{T}^{ \pm(i j) \pm} \mathbf{T}\left(\mathbf{k}_{s}, \pm k_{z i}, \mathbf{r}\right) \\
& \otimes \mathbf{T}^{*}\left(\mathbf{k}_{s}, \pm k_{z j}, \mathbf{s}\right) .
\end{aligned}
$$

A summation is implied for each pair of \pm indices. These indices show the direction of propagation of the electromagnetic modes, the first index for the acceptor and the second for the donor. Also the summation over $\mathbf{T}$ is over the $\mathbf{M}$ and $\mathbf{N}$ modes which are connected with the transverse electric and transverse magnetic modes, respectively. The form of $\mathbf{M}$ and $\mathbf{N}$ can be found in Ref. [52]. For planar geometries there are no hybrid modes. The boundary conditions imposed on the system of multilayers are the continuity condition and the radiation condition. The first condition is given by continuity equations at each interface:

$$
\begin{gathered}
\hat{\mathbf{z}} \times\left.\left[\mathfrak{G}^{(i j)}(\mathbf{r}, \mathbf{s}, \omega)-\mathfrak{G}^{(i+1) j)}(\mathbf{r}, \mathbf{s}, \omega)\right]\right|_{z=d_{i}}=0, \\
\hat{\mathbf{z}} \times\left.\left[\nabla \times \mathfrak{G}^{(i j)}(\mathbf{r}, \mathbf{s}, \omega)-\nabla \times \mathfrak{G}^{((i+1) j)}(\mathbf{r}, \mathbf{s}, \omega)\right]\right|_{z=d_{i}}=-i \frac{4 \pi}{c} k_{0} \sigma \hat{z} \times \hat{z} \times \mathfrak{G}^{(i+1) j)}(\mathbf{r}, \mathbf{s}, \omega),
\end{gathered}
$$

where $\sigma$ is the surface conductivity of the twodimensional material; for our case it is the $\mathrm{MoS}_{2}$ layer, Eq. (7).

By applying these boundary equations, an inhomogeneous system of $2^{N-1}$ equations is defined which have $2^{N-1}$ unknowns, the generalized $R_{M(N)}^{ \pm(i j) \pm}$ coefficients. These coefficients are sufficient to uniquely determine the problem under consideration through the exact knowledge of the scattering part of the Green's tensor. In order to find the generalized coefficients, a matrix equation is solved which has the form

$$
\Delta_{M(N)} \cdot \boldsymbol{R}_{M(N)}^{(i) \pm}=\boldsymbol{V}_{M(N)}^{(i) \pm},
$$

where $\Delta$ is the characteristic matrix of the system of equations from the boundary conditions at the interfaces, $\boldsymbol{R}^{(i) \pm}$ is the column of the generalized coefficients $R_{M(N)}^{ \pm(i j) \pm}$, and $\boldsymbol{V}^{(i) \pm}$ is the free term vector whose terms are given by the homogeneous part of the Green's tensor. 
We will consider in more detail the case where a 2D material $\mathrm{MoS}_{2}$ is sandwiched between two planar half spaces with dielectric permittivities $\varepsilon_{1}$ and $\varepsilon_{2}$. The $z$ direction is perpendicular to the boundary between the two half spaces [52,53]. Using Eq. (A1) the Green's tensor has the form

$$
\begin{aligned}
\mathfrak{G}^{(11)}(\mathbf{r}, \mathbf{s}, \omega) & =\mathfrak{G}_{h}^{(11)}(\mathbf{r}, \mathbf{s}, \omega)+\mathfrak{G}_{s}^{(11)}(\mathbf{r}, \mathbf{s}, \omega), \\
\mathfrak{G}^{(21)}(\mathbf{r}, \mathbf{s}, \omega) & =\mathfrak{G}_{s}^{(21)}(\mathbf{r}, \mathbf{s}, \omega),
\end{aligned}
$$

where the first of the two labels in the superscript $(i 1)$ denotes the field point, while the second denotes the source point. The scattering terms have the following expression

$$
\begin{aligned}
\mathfrak{G}_{s}^{(11)}(\mathbf{r}, \mathbf{s}, \omega)= & \frac{i}{8 \pi^{2}} \sum_{K} \int \mathrm{d}^{2} k_{s} \frac{1}{k_{z 1} k_{s}^{2}} R_{K}^{+11-} \mathbf{K}\left(k_{s}, k_{z 1}, \mathbf{r}\right) \\
& \otimes \mathbf{K}^{*}\left(k_{s},-k_{z 1}, \mathbf{s}\right) \\
\mathfrak{G}_{s}^{(21)}(\mathbf{r}, \mathbf{s}, \omega)= & \frac{i}{8 \pi^{2}} \sum_{K} \int \mathrm{d}^{2} k_{s} \frac{1}{k_{z 1} k_{s}^{2}} R_{K}^{-21-} \mathbf{K}\left(k_{s},-k_{z 2}, \mathbf{r}\right) \\
& \otimes \mathbf{K}^{*}\left(k_{s},-k_{z 1}, \mathbf{s}\right),
\end{aligned}
$$

where $k_{s}=\sqrt{k_{i}^{2}-k_{z i}^{2}}$ is the in-plane propagation constant, $k_{z i}$ is the perpendicular propagation constant in medium $i$, and $k_{i}=\frac{\omega}{c} \sqrt{\varepsilon_{i}}$ is the wave number in medium $i(i=1,2)$. The above expressions involve a summation over $\mathbf{K}$ which represents $\mathbf{M}$ and $\mathbf{N}$, the transverse electric (TE) and transverse magnetic (TM) modes, respectively.

Imposing the continuity conditions, Eq. (A5), at the boundary between the two half spaces, $z=0$, we obtain the generalized Fresnel coefficients, which have the form $[35,36]$

$$
\begin{aligned}
& R_{M}^{11}=\frac{k_{z 1}-k_{z 2}-2 \alpha k_{0}}{k_{z 1}+k_{z 2}+2 \alpha k_{0}}, \quad R_{N}^{11}=\frac{k_{2}^{2} k_{z 1}-k_{1}^{2} k_{z 2}+2 \alpha k_{0} k_{z 1} k_{z 2}}{k_{2}^{2} k_{z 1}+k_{1}^{2} k_{z 2}+2 \alpha k_{0} k_{z 1} k_{z 2}} \\
& R_{M}^{21}=\frac{2 k_{z 1}}{k_{z 1}+k_{z 2}+2 \alpha k_{0}}, \quad R_{N}^{21}=\frac{2 k_{1} k_{2} k_{z 1}}{k_{2}^{2} k_{z 1}+k_{1}^{2} k_{z 2}+2 \alpha k_{0} k_{z 1} k_{z 2}},
\end{aligned}
$$

where $\alpha=2 \pi \sigma / c$.
[1] E. M. Purcell, Phys. Rev. 69, 681 (1946).

[2] S. A. Maier and H. A. Atwater, J. Appl. Phys. 98, 011101 (2005).

[3] C. A. Marocico and J. Knoester, Phys. Rev. A 84, 053824 (2011).

[4] J. B. Khurgin, Nat. Nanotechnol. 10, 2 (2015).

[5] T. Low and P. Avouris, ACS Nano 8, 1086 (2014).

[6] F. J. García de Abajo, ACS Photonics 1, 135 (2014).

[7] P. Avouris, Nano Lett. 10, 4285 (2010).

[8] L. Gaudreau, K. J. Tielrooij, G. E. D. K. Prawiroatmodjo, J. Osmond, F. J. G. de Abajo, and F. H. L. Koppens, Nano Lett. 13, 2030 (2013)

[9] J. Lee, W. Bao, L. Ju, P. J. Schuck, F. Wang, and A. WeberBargioni, Nano Lett. 14, 7115 (2014).

[10] F. Yang, J. R. Sambles, and G. W. Bradberry, Phys. Rev. Lett. 64, 559 (1990).

[11] J. D. Caldwell, L. Lindsay, V. Giannini, I. Vurgaftman, T. L. Reinecke, S. A. Maier, and O. J. Glembocki, Nanophotonics 4, 1 (2015).

[12] A. Scholz, T. Stauber, and J. Schliemann, Phys. Rev. B 88, 035135 (2013).

[13] Y. N. Gartstein, X. Li, and C. Zhang, Phys. Rev. B 92, 075445 (2015).

[14] J. B. Khurgin, Optica 2, 740 (2015).

[15] L. Britnell, R. M. Ribeiro, A. Eckmann, R. Jalil, B. D. Belle, A. Mishchenko, Y.-J. Kim, R. V. Gorbachev, T. Georgiou, S. V. Morozov, A. N. Grigorenko, A. K. Geim, C. Casiraghi, A. H. C. Neto, and K. S. Novoselov, Science 340, 1311 (2013).

[16] F. Xia, H. Wang, D. Xiao, M. Dubey, and A. Ramasubramaniam, Nat. Photon. 8, 899 (2014).

[17] D. Xiao, G. B. Liu, W. Feng, X. Xu, and W. Yao, Phys. Rev. Lett. 108, 196802 (2012).

[18] H. Yu, G.-B. Liu, P. Gong, X. Xu, and W. Yao, Nat. Commun. 5, 3876 (2014).

[19] O. Lopez-Sanchez, D. Lembke, M. Kayci, A. Radenovic, and A. Kis, Nat. Nanotechnol. 8, 497 (2013).
[20] D. Kozawa, R. Kumar, A. Carvalho, K. Kumar Amara, W. Zhao, S. Wang, M. Toh, R. M. Ribeiro, A. H. Castro Neto, K. Matsuda, and G. Eda, Nat. Commun. 5, 4543 (2014).

[21] C. Chakraborty, L. Kinnischtzke, K. M. Goodfellow, R. Beams, and A. N. Vamivakas, Nat. Nanotechnol. 10, 507 (2015).

[22] M. Amani, D.-H. Lien, D. Kiriya, J. Xiao, A. Azcatl, J. Noh, S. R. Madhvapathy, R. Addou, S. KC, M. Dubey, K. Cho, R. M. Wallace, S.-C. Lee, J.-H. He, J. W. Ager, X. Zhang, E. Yablonovitch, and A. Javey, Science 350, 1065 (2015).

[23] K. Messer, M. Eggleston, S. B. Desai, S. A. Fortuna, S Madhavapathy, P. Zhao, J. Xiao, X. Zhang, A. Javey, M. C. $\mathrm{Wu}$, and E. Yablonovitch, 2015 Fourth Berkeley Symposium on Energy Efficient Electronic Systems (E3S) (IEEE, Piscataway, NJ, 2015).

[24] M. I. Vasilevskiy, D. G. Santiago-Pérez, C. Trallero-Giner, N. M. R. Peres, and A. Kavokin, Phys. Rev. B 92, 245435 (2015).

[25] B. Radisavljevic, A. Radenovic, J. Brivio, V. Giacometti, and A. Kis, Nat. Nanotechnol. 6, 147 (2011).

[26] F. Prins, A. J. Goodman, and W. A. Tisdale, Nano Lett. 14, 6087 (2014).

[27] D. Kufer, I. Nikitskiy, T. Lasanta, G. Navickaite, F. H. L. Koppens, and G. Konstantatos, Adv. Mater. 27, 176 (2015).

[28] S. Sampat, T. Guo, K. Zhang, J. A. Robinson, Y. Ghosh, K. P. Acharya, H. Htoon, J. A. Hollingsworth, Y. N. Gartstein, and A. V. Malko, ACS Photonics 3, 708 (2016).

[29] H. Zang, P. K. Routh, Y. Huang, J.-S. Chen, E. Sutter, P. Sutter, and M. Cotlet, ACS Nano 10, 4790 (2016).

[30] A. Raja, A. Montoya-Castillo, J. Zultak, X.-X. Zhang, Z. Ye, C. Roquelet, D. A. Chenet, A. M. van der Zande, P. Huang, S. Jockusch, J. Hone, D. R. Reichman, L. E. Brus, and T. F. Heinz, Nano Lett. 16, 2328 (2016).

[31] K. M. Goodfellow, C. Chakraborty, K. Sowers, P. Waduge, M. Wanunu, T. Krauss, K. Driscoll, and A. N. Vamivakas, Appl. Phys. Lett. 108, 021101 (2016). 
[32] C. Zhang, H. Wang, W. Chan, C. Manolatou, and F. Rana, Phys. Rev. B 89, 205436 (2014).

[33] H. T. Dung, L. Knöll, and D.-G. Welsch, Phys. Rev. A 57, 3931 (1998).

[34] H. T. Dung, L. Knöll, and D.-G. Welsch, Phys. Rev. A 62, 053804 (2000).

[35] G. W. Hanson, J. Appl. Phys. 103, 064302 (2008).

[36] A. Y. Nikitin, F. J. Garcia-Vidal, and L. Martin-Moreno, IEEE J. Sel. Top. Quantum Electron. 19, 4600611 (2013).

[37] H. Dery and Y. Song, Phys. Rev. B 92, 125431 (2015).

[38] M. Palummo, M. Bernardi, and J. C. Grossman, Nano Lett. 15, 2794 (2015).

[39] T. Stauber, D. Noriega-Pérez, and J. Schliemann, Phys. Rev. B 91, 115407 (2015).

[40] S. A. Mikhailov and K. Ziegler, Phys. Rev. Lett. 99, 016803 (2007).

[41] G. W. Hanson, S. A. Hassani Gangaraj, C. Lee, D. G. Angelakis, and M. Tame, Phys. Rev. A 92, 013828 (2015).

[42] V. D. Karanikolas, C. A. Marocico, and A. L. Bradley, Phys. Rev. B 91, 125422 (2015).
[43] J. A. Dionne, L. A. Sweatlock, H. A. Atwater, and A. Polman, Phys. Rev. B 72, 075405 (2005).

[44] V. Agranovich, H. Benisty, and C. Weisbuch, Solid State Commun. 102, 631 (1997).

[45] G. W. Ford and W. H. Weber, Phys. Rep. 113, 195 (1984).

[46] J. M. Gordon and Y. N. Gartstein, J. Phys.: Condens. Matter 25, 425302 (2013).

[47] H. Wang, H. Feng, and J. Li, Small 10, 2165 (2014).

[48] S.-G. Yi, J. H. Kim, J. K. Min, M. J. Park, Y. W. Chang, and K.-H. Yoo, IEEE Trans. Nanotech. 15, 499 (2016).

[49] T. Christensen, W. Yan, A.-P. Jauho, M. Wubs, and N. A. Mortensen, Phys. Rev. B 92, 121407 (2015).

[50] V. D. Karanikolas, C. A. Marocico, and A. L. Bradley, Phys. Rev. B 93, 035426 (2016).

[51] C. T. Tai, Dyadic Green Functions in Electromagnetic Theory (IEEE Press, New York, NY, 1994).

[52] W. C. Chew, Waves and Fields in Inhomogeneous Media (IEEE Press, New York, NY, 1995).

[53] L. Novotny and B. Hecht, Principles of nano-optics, 2nd ed. (Cambridge University Press, Cambridge, UK, 2012). 